\title{
An adaptive LTE listen-before-talk scheme towards a fair coexistence with Wi-Fi in unlicensed spectrum
}

\author{
Vasilis Maglogiannis $^{1} \mathbb{D} \cdot$ Dries Naudts $^{1} \cdot$ Adnan Shahid $^{1} \cdot$ Ingrid Moerman $^{1}$
}

Published online: 10 January 2018

(c) The Author(s) 2018. This article is an open access publication

\begin{abstract}
The technological growth combined with the exponential increase of wireless traffic are pushing the wireless community to investigate solutions to maximally exploit the available spectrum. Among the proposed solutions, the operation of Long Term Evolution (LTE) in the unlicensed spectrum (LTE-U) has attracted significant attention. Recently, the 3rd Generation Partnership Project announced specifications that allow LTE to transmit in the unlicensed spectrum using a Listen Before Talk (LBT) procedure, respecting this way the regulator requirements worldwide. However, the proposed standards may cause coexistence issues between LTE and legacy Wi-Fi networks. In this article, it is discussed that a fair coexistence mechanism is needed to guarantee equal channel access opportunities for the co-located networks in a technology-agnostic way, taking into account potential traffic requirements. In order to enable harmonious coexistence and fair spectrum sharing among LTE-U and Wi-Fi, an adaptive LTE-U LBT scheme is presented. This scheme uses a variable LTE transmission opportunity (TXOP) followed by a variable muting period. This way, co-located Wi-Fi networks can exploit the muting period to gain access to the wireless medium. The scheme is studied and evaluated in different compelling scenarios using a simulation platform. The results show that by configuring the LTE-U with the appropriate TXOP and muting period values, the proposed scheme can significantly improve the coexistence among LTE-U and Wi-Fi in a fair manner. Finally, a preliminary algorithm is proposed on how the optimal configuration parameters can be selected towards harmonious and fair coexistence.
\end{abstract}

Keywords LTE $\cdot$ Wi-Fi $\cdot$ LTE unlicensed $\cdot$ LTE LAA $\cdot$ Transmission opportunity $\cdot$ Muting period $\cdot$ Fairness

\section{Introduction}

Over the last years, the technological growth has led to a tremendous increase of wireless devices such as smartphones, tablets, laptops and wearable technologies. Additionally, the number of electronic devices that exchange information wirelessly is growing day by day, pushed by the evolution and consolidation of the Internet of Things (IoT).

$凶$ Vasilis Maglogiannis

vasilis.maglogiannis@ugent.be

Dries Naudts

dries.naudts@ugent.be

Adnan Shahid

adnan.shahid@ugent.be

Ingrid Moerman

ingrid.moerman@ugent.be

1 IDLab, Department of Information Technology, Ghent University - imec, Technologiepark-Zwijnaarde 15, 9052 Ghent, Belgium
According to Qualcomm, the amount of wireless traffic is expected to further increase by a factor of 1000 by 2020 [1]. This massive amount of information is exchanged between devices using different types of technologies such as Long Term Evolution (LTE), IEEE 802.11, IEEE 802.15.4 and Bluetooth. Lately, sub-gigahertz bands are exploited by technologies like LORA and SIGFOX in order to achieve wide range communications. Additionally, high frequency bands such as mmWave are used for multi-gigabit speeds (IEEE 802.11ad). It becomes clear that the wireless network capacity will soon become a bottleneck for the increased wireless traffic.

Concurrently, the licensed spectrum used by the mobile operators becomes very scarce. The limitation of the licensed spectrum in combination with the high cost of a licensed frequency band have pushed the mobile operators to investigate other technological solutions that can support in meeting the 1000x challenge requirements. These solutions include among others, enhanced massive Multiple-Input MultipleOutput (MIMO), Carrier Aggregation, cloud computing 
services, as well as LTE operation in the unlicensed spectrum (LTE-U). Among various other solutions, the last one has attracted significant attention from the wireless community. Several mechanisms, such as Listen Before Talk (LBT) have been proposed towards the coexistence of LTE-U and other well-established technologies in the unlicensed spectrum, such as Wi-Fi [2].

In markets like the U.S., China and South Korea where a Clear Channel Assessment (CCA) mechanism (also known as LBT) is not required, LTE can operate in the unlicensed spectrum using techniques such as Carrier Sense Adaptive Transmission (CSAT) [3]. CSAT, proposed by Qualcomm, exploits duty cycle periods in order to give to Wi-Fi transmission opportunities (TXOP). It divides the time into LTE ON and LTE OFF slots. During an ON period, LTE transmits without sensing the medium, while during an OFF period it remains silent giving TXOP to other coexisting networks.

Recently, key players of the mobile world have proposed standards to the 3rd Generation Partnership Project (3GPP), which specify the LTE operation in unlicensed spectrum. 3GPP announced the operation of LTE Licensed-Assisted Access (LTE LAA) [4], as an enhancement towards 3GPP LTE Release 13. LTE LAA allows operators to transmit in the unlicensed spectrum using a secondary cell, alongside a primary cell operating in the licensed band that they own. Using LTE LAA, a mobile operator can offload the LTE network into the unlicensed spectrum, when this is required. The licensed spectrum can be used to ensure the transmission of the crucial LTE control signals without interference. Additionally, applications that require high Quality of Service (QoS) (e.g. video streaming) can exploit the advantages of the interference-free licensed spectrum. LTE LAA requires a CCA procedure before each transmission in the unlicensed spectrum. This way, the mechanism can be applicable worldwide, including markets like Europe and Japan, where CCA is mandatory.

In order to decouple LTE from the operators, leading wireless stakeholders proposed the LTE operation solely in the unlicensed spectrum as a standalone wireless solution. To this end, they formed the MulteFire Alliance [5]. Hence, LTE can be deployed by Internet Service Providers (ISPs), building owners, cable companies, etc. The underlying technique proposed by the alliance builds on elements of 3GPP LTE LAA.

Although the LTE LAA standard defines that a CCA procedure must be performed before a transmission burst, it also defines four different channel access priority classes. Each channel access priority class specifies among others the duration of the transmission burst that follows a successful CCA procedure. This duration ranges from 2 to $10 \mathrm{~ms}$ [2]. On the contrary, a Wi-Fi packet transmission when frame aggregation is not enabled or supported by the 802.11 standard typically lasts a few hundreds of $\mu \mathrm{s}$ [6]. Furthermore in [7], it has been assessed that for $802.11 \mathrm{n}$ with frame aggregation, $50 \%$ of the packets are transmitted within $30 \mu \mathrm{s}$, while $80 \%$ of the packets are transmitted within $1 \mathrm{~ms}$. It is clear that the ratio between LTE and Wi-Fi channel occupancy is not balanced. This can result to unfair coexistence between co-located LTE LAA and Wi-Fi networks.

In this article, we discuss a way that fairness can be achieved between LTE-U and Wi-Fi. We define a new adaptive LTE-U transmission scheme according to which LTE can transmit in unlicensed spectrum using a variable TXOP time. This TXOP period is followed by a variable muting period in order to give channel access opportunities to other potentially co-located networks, such as Wi-Fi. Before a TXOP, LTE must perform a CCA procedure in order to determine the availability of the channel. This scheme can be used for LTE transmissions in the unlicensed spectrum next to the primary cell that an operator uses in the licensed spectrum similar to LTE LAA. The proposed scheme is evaluated through simulations. Finally, we discuss how the configurations of TXOP and muting period can be selected by a network in order to provide fair coexistence. The main contribution of this work is summarized as follows:

- Discussion about fairness and definition of fairness as equal sharing of the wireless resources in a technologyagnostic manner

- Verification of the problem by evaluating the coexistence between LTE LAA and Wi-Fi, when Wi-Fi operates in a traditional way, meaning that it does not support or it does not use frame aggregation

- Proposal of a new adaptive LTE-U transmission scheme that uses a variable TXOP followed by a variable muting period. The proposed scheme performs a channel estimation before a transmission to ensure the availability of the channel

- Discussion about the selection of the TXOP and muting period combinations that can offer fair coexistence between LTE-U and Wi-Fi

The remainder of the article is organized as follows. In Sect. 2 we present an overview of the main characteristics of LTE and Wi-Fi that lead to coexistence issues when the two technologies operate next to each other in their traditional form. Section 3 discusses the current literature on LTE-U and especially LTE LAA. Section 4 defines the problem that arises when LTE LAA coexists with traditional Wi-Fi networks that do not use frame aggregation and describes the proposed solution. Next, in Sect. 5 we discuss about fair coexistence in unlicensed spectrum and the approach that we follow in this article. Section 6 presents the simulation platform that has been used. In Sect. 7, we discuss the simulation scenarios that are studied. Then, in Sect. 8, we present and discuss the obtained results for each investigated scenario. 


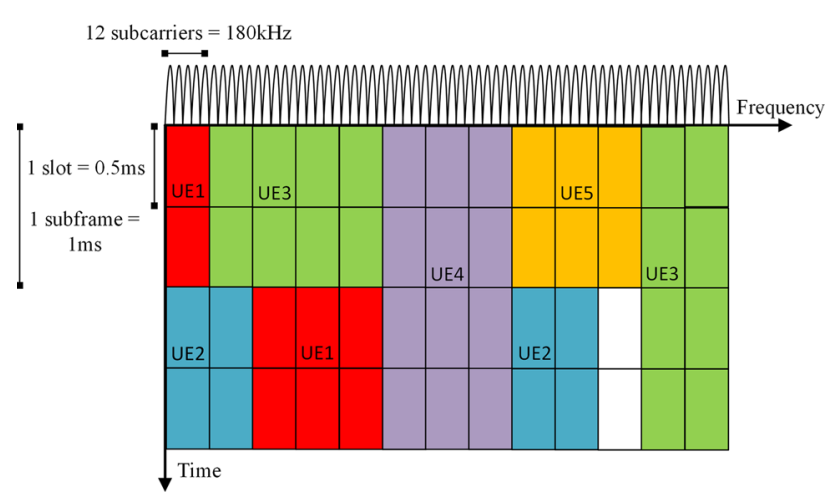

Fig. 1 LTE time-frequency structure and scheduling of different UEs

In Sect. 9, we discuss the way that a selection of the configuration parameters can be done for the proposed scheme towards fair coexistence. Finally, in Sect. 10, we conclude the paper and discuss plans for future work.

\section{LTE and Wi-Fi in a nutshell}

This section briefly discusses the main differences between LTE and Wi-Fi that result in unfair coexistence, when the two technologies operate in the proximity of each other in their traditional form.

LTE uses multi-user versions of the Orthogonal Frequency Division Multiplexing (OFDM) digital modulation scheme, called Orthogonal Frequency Division Multiple Access (OFDMA) for the DL and Single-Carrier Frequency Division Multiple Access (SC-FDMA) for the UL [8]. LTE divides the time domain in time slots of $0.5 \mathrm{~ms}$ duration. Each time slot corresponds to 7 OFDM symbols when Normal Cyclic Prefix (CP) is used and to 6 OFDM symbols for Extended CP. An LTE radio frame has a duration of $10 \mathrm{~ms}$ and consists of 10 subframes. Each subframe consists of 2 slots. The frequency domain is divided into subcarriers each one occupying $15 \mathrm{KHz}$ of bandwidth. Combining one time slot and 12 subcarriers, LTE defines the Resource Block (RB), which is the unit of transmission.

LTE is a scheduled technology designed to operate in the licensed spectrum. Hence, it does not require to sense the medium before a transmission. The LTE base station named Evolved NodeB (eNB) schedules the different User Equipment (UE) transmissions on a subframe basis, meaning that every $1 \mathrm{~ms}$ the assignment of the subframes to the active UE can change. The time-frequency structure and the assignment of the resources to different UEs is depicted in Fig. 1.

On the other hand, Wi-Fi uses OFDM digital modulation scheme and the Distributed Coordination Function (DCF) as the fundamental mechanism to access the wireless medium, which is designed to be asynchronous and decentralized [6]. Wi-Fi uses a Carrier Sensing Multiple Access with Colli-

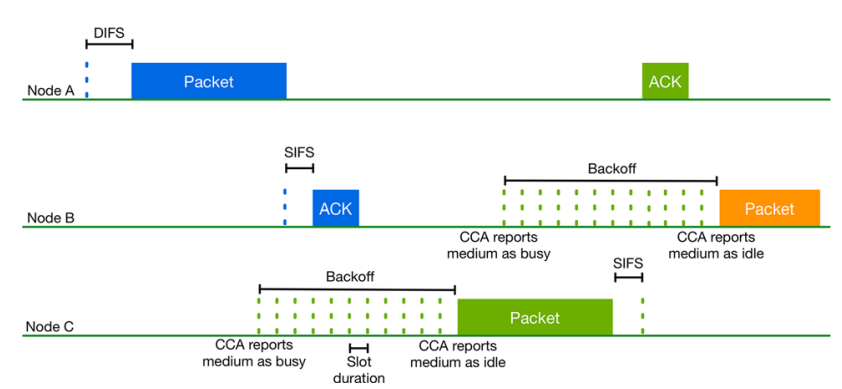

Fig. 2 802.11 CSMA/CA procedure

sion Avoidance (CSMA/CA) mechanism to compete for the channel access. Before a transmission, Wi-Fi has to sense the medium to determine if it is busy or idle This procedure is known as Clear Channel Assessment (CCA). Figure 2 illustrates the CSMA/CA procedure. Only if the medium is idle for DCF Inter-Frame Space (DIFS) the node can transmit. Otherwise and also prior to a new transmission immediately after a successful transmission, the node has to postpone its transmission for DIFS plus a random backoff time. The backoff time indicates how many idle time slots a node has to sense before a transmission. The number of the timeslots is specified by the backoff counter that is randomly selected within the range of the Contention Window (CW). If the transmission is not successful and an acknowledgement (ACK) is not received the node schedules a retransmission after a new exponential backoff period until the maximum number of retransmissions is reached.

CCA consists of two functions named Carrier Sense (CS) and Energy Detection (ED). The CS function refers to the ability of the receiver to detect and decode a received $\mathrm{Wi}-\mathrm{Fi}$ preamble. The ED function is used when the received signal cannot be decoded. According to the standards the threshold of CS and ED for $20 \mathrm{MHz}$ bandwidth is -82 and $-62 \mathrm{dBm}$ respectively [6].

\section{Related work}

In our previous work [9], we studied the impact of traditional LTE operating in unlicensed spectrum on Wi-Fi using OffThe-Shelf (OTS) hardware equipment using the LTE testbed of IMEC [10]. In this study, three different levels of LTE signal have been examined, representing different possible levels of LTE impact on Wi-Fi. The results show that the Wi-Fi performance, in terms of throughput and latency, can be significantly affected by LTE. In [11], the authors performed an experimental evaluation to study the impact of LTE LAA on Wi-Fi performance in indoor office environment. The study includes analysis of LTE LAA interference for five different scenarios. Based on this analysis the authors provide LTE LAA Medium Access Control (MAC) designs 
to deal with coexistence issues with Wi-Fi. Several other studies [12-14] evaluate the Wi-Fi performance degradation based on mathematical models and simulations. All studies come to the same conclusion, namely that coexistence mechanisms are required to enable coexistence between co-located LTE and Wi-Fi networks.

The authors in [15] evaluate through simulations the performance impact of LTE and Wi-Fi when both networks operate in the same frequency. They propose a coexistence mechanism similar to CSAT that exploits periodically blank LTE subframes during an LTE frame in order to give opportunity to Wi-Fi to transmit. They conclude that the network topology, as well as the number and order of the blank subframes lead to different coexistence results.

Towards a global coexistence technique that respects the regional regulations, 3GPP announced the LTE LAA standards in Release 13, including the description of a CCA procedure [2]. Initially, LTE LAA is scheduled to operate for DL only and within the $5 \mathrm{GHz}$ channel. Towards Release 14 , it is expected to be extended to $2.4 \mathrm{GHz}$ unlicensed band and for both DL and UL traffic. The transmission in the unlicensed spectrum can be done via a secondary cell operating alongside the primary cell owned by the operator. This feature can be enabled using the Carrier Aggregation mechanism that has been introduced in 3GPP LTE Release 10 [16].

In [17], the authors provide a description of the LTE LAA mechanisms including motivation and use cases to which it can be applied. They present a coexistence evaluation methodology and results, which have been contributed by 3GPP.

The authors of [18] present a detailed overview of LTE LAA in Release 13. They show how the introduction of CCA and the discontinuous transmission impose changes in different LTE components such as the DL physical channels, the hybrid automatic repeat request (HARQ) feedback procedures, etc. Simulation results are presented to show that coexistence with Wi-Fi can be enabled in a range of scenarios. Moreover, an overview of LTE LAA enhancements beyond Release 13 is given.

In [19] two non-coordinated and two coordinated network management approaches to enable coexistence are proposed. Regarding the non-coordinated techniques, the first one proposes eNB to perform CCA on different channels and to switch to a different channel after a transmission, while the second proposes LTE to offer transmission opportunities of variable duration to Wi-Fi after a transmission based on the occupancy of the medium. Concerning the coordinated methodologies, the first one proposes a Network Function Virtualization (NFV) interconnection to combine the Wi-Fi network and the LTE-U service provider. Channel selection and seamless transfer of resources between the two technologies can be enabled, using the in-the-cloud control of distributed Access Points (APs). The second method pro- poses the management of coexistence using the $\mathrm{X} 2$ interface among the eNBs. The eNBs can exchange information and schedule Almost Blank Subframes (ABS) in different subframes giving this way more opportunities to any $\mathrm{Wi}-\mathrm{Fi}$ network that is located potentially within their proximity. In the aforementioned schemes, the different Radio Access Technologies (RATs) are under the control of the same mobile operator.

The authors in [20] propose an LBT protocol for LTE LAA that enhances the coexistence with Wi-Fi and increases the overall system performance. This LBT scheme consists of two different mechanisms named on-off adaptation for channel occupancy time and short-long adaptation for idle time. The first mechanism is responsible to adapt the channel occupancy time of LTE based on the load of the network, while the second one adapts the idle period based on the $\mathrm{CW}$ duration of Wi-Fi.

In [21], the authors propose an LBT mechanism for LTE LAA that aims to share the medium in a fair way towards the increase of the overall system performance. The mathematical analysis of the proposed LBT scheme is validated via simulations. The results show that a proper selection of LAA channel occupancy and backoff counter can increase the performance of Wi-Fi.

In [22], the coexistence between LTE LAA and Wi-Fi is studied using LBT category 4 channel access scheme. The behaviour of LAA eNB is modelled as a Markov Chain and the obtained throughput is adopted as performance metric. The proposed LBT scheme uses an adaptive CW size for LTE LAA. According to the results, the proposed scheme outperforms the fixed CW size.

The authors in [23] describe and evaluate a channel switch function that is used to determine the LTE LAA channel dynamically. This way, LTE LAA can exploit the spectrum in a more flexible way. They propose an enhanced LBT scheme with channel switch that uses a frozen period to select the appropriate channel. The channel switch is done based on a proportional fair based dynamic channel switch method that is analytically presented. The results show that the proposed scheme can increase the overall system performance.

In [24], a MAC layer for LTE-U is proposed that uses an LBT algorithm and channel reservation packets. Both synchronous and asynchronous LBT are examined. Additionally, improvements to the LTE link adaptation algorithm are proposed in order to cope with potential collisions. Simulation results indicate that the performance of $\mathrm{Wi}-\mathrm{Fi}$ can be improved by the proposed MAC design. Furthermore, the channel reservation mechanisms increase the LTE-U cell edge performance.

In our previous work [25], we extensively studied the concept of LTE-U. Initially, we provide a detailed analysis of the current state-of-the-art regarding LTE-U and Wi-Fi. Furthermore, the article presents a classification of techniques 
Table 1 LTE LAA channel access priority class configurations

\begin{tabular}{llllll}
\hline Channel access priority class (p) & $\mathrm{m}_{\mathrm{p}}$ & $\mathrm{CW}_{\text {min, } \mathrm{p}}$ & $\mathrm{CW}_{\text {max }, \mathrm{p}}$ & $\mathrm{T}_{\text {mcot } \mathrm{p}}(m s)$ & Allowed $\mathrm{CW}_{\mathrm{p}}$ sizes \\
\hline 1 & 1 & 3 & 7 & 2 & 3,7 \\
2 & 1 & 7 & 15 & 3 & 7,15 \\
3 & 3 & 15 & 63 & 8 or 10 & $15,31,63$ \\
4 & 7 & 15 & 1023 & 8 or 10 & $15,31,63,127,255,511,1023$ \\
\hline
\end{tabular}

that can be applied between co-located LTE and Wi-Fi networks. This classification in combination with the study of the literature revealed the lack of cooperation schemes among co-located networks that can lead to more optimal use of the available spectrum. In order to fill this gap, several concepts of cooperation techniques that can enhance the spectral efficiency between coexisting LTE and Wi-Fi networks are proposed. Additionally, the proposed cooperation schemes are compared between each other in terms of complexity and performance.

Finally, the authors in [26] provide a detailed survey of the coexistence of LTE-U and Wi-Fi on $5 \mathrm{GHz}$ with the corresponding deployment scenarios. They provide a detailed description of the coexistence-related features of LTE-U and $\mathrm{Wi}-\mathrm{Fi}$, the coexistence challenges, the differences in performance between the two different technologies and co-channel interference. They extensively discuss the proposed coexistence mechanisms between LTE-U and Wi-Fi in the current literature. Furthermore, the survey discusses the concept of the scenario-oriented coexistence, in which coexistence-related problems are solved according to different deployment scenarios.

Although the 3GPP standards specify that the channel must be sensed by a CCA procedure before a transmission, the ratio between LTE LAA and Wi-Fi transmission opportunities is not balanced, especially in the case that Wi-Fi does not support or use frame aggregation. According to the best of our knowledge, the current literature lacks of a mechanism that can adapt the LTE-U channel access after a CCA in order to provide equal channel opportunities to other co-located networks such as Wi-Fi. The following aspects render our proposal novel and valuable. Firstly, the proposed scheme is flexible as it adapts the LTE-U channel access in order to provide fair coexistence with networks in unlicensed spectrum based on various parameters such as the number of the colocated networks and the type of traffic that has to be served (e.g. delay-sensitive traffic). Secondly, the CCA procedure ensures that the mechanism can be applicable worldwide. Thirdly, this scheme can provide fair coexistence not only to Wi-Fi but also to other well-established technologies in unlicensed spectrum, such as 802.15.4 and Bluetooth. Finally, the proposed variable TXOP followed by a variable muting period does not have an impact on time-sensitive LTE traffic, as it can still be transmitted via the licensed band of the operator.

\section{Problem definition and proposal description}

Recently, 3GPP announced the LTE LAA standards as part of LTE Release 13. LTE LAA defines that a CCA procedure [2] must be performed before an LTE transmission in the unlicensed spectrum. This way, the standard can be applicable worldwide, as it respects the regional regulations in markets like Europe and Japan where a CCA procedure is mandatory.

Initially, LTE LAA (as defined in Release 13) is scheduled to operate within the $5 \mathrm{GHz}$ unlicensed spectrum and for Downlink (DL) traffic only, while the Uplink (UL) traffic will be maintained in the licensed spectrum. In a later phase towards Release 14, it is expected to be extended to $2.4 \mathrm{GHz}$ unlicensed band including both DL and UL traffic. According to LTE LAA Release 13, an eNB will be able to activate and deactivate a secondary cell operating in the unlicensed spectrum. Via this cell only DL data traffic can be sent through the Physical DL Shared Channel (PDSCH). The LTE control signals and the UL traffic will be maintained in the licensed anchor via the Physical UL Shared Channel (PUSCH). Especially for the LTE control signals whose transmission is time-critical, the licensed anchor can guarantee a safe and interference-free transmission.

Before a transmission, an eNB must perform the CCA procedure in order to sense the channel in the unlicensed spectrum. When the channel is sensed as busy, the eNB must defer its transmission and perform an exponential backoff. If the medium is sensed as idle, the eNB starts a transmission burst with a duration varying form 2 up to $10 \mathrm{~ms}$, depending on selected channel access priority class. Table 1 shows the definitions of the different channel access priority classes. The smaller the number of the class, the higher the priority.

From the table, it can be seen that each priority class uses different $T_{\text {mcot,p }}$ that refers to the maximum channel occupancy time for the specific class $\mathrm{p}$. According to the standard, for the priority classes 3 and 4 , the $T_{m c o t, p}$ equals to $10 \mathrm{~ms}$ if the absence of any other co-located unlicensed technology sharing the same spectrum band can be guaranteed on a long term basis. In a different case, it is limited to $8 \mathrm{~ms}$. An eNB cannot continuously transmit in unlicensed spectrum for a period longer than $T_{m c o t, p}$. After the end of the $\mathrm{T}_{\text {mcot.p }}$, it must perform a CCA procedure to estimate again the occupancy of the channel. 


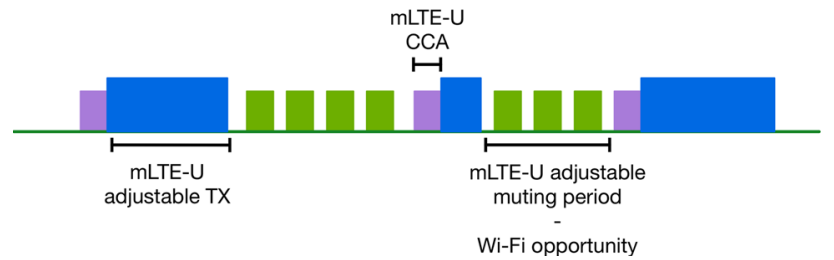

Fig. 3 The design of the proposed mLTE-U scheme

On the other hand, in traditional Wi-Fi network without frame aggregation, an AP or a Station (STA) transmits only one packet after it successfully estimates the medium as idle. Such a Wi-Fi packet transmission typically lasts a few hundreds of $\mu \mathrm{s}$. After the transmission of the packet, it has to compete again to access the medium against other co-located networks by performing a CCA procedure. In several still widely used Wi-Fi standards such as $802.11 \mathrm{a} / \mathrm{g}$ frame aggregation is not supported. Even if frame aggregation is available (e.g. 802.11n/ac [27]), often it is not used depending on the traffic type (e.g. low latency constraints) [28].

It is clear that the transmission durations of LTE LAA and Wi-Fi are not balanced as the TXOP duration of LTE LAA is significantly longer compared to a single packet transmission of Wi-Fi. Moreover, as both networks perform an exponential backoff after they sense the channel as busy, it is possible for an LTE LAA network to gain consecutive times access to the channel forcing Wi-Fi to postpone its transmission for even longer period of time. This can lead to unfair coexistence between co-located LTE and Wi-Fi networks. Especially in the case of multiple LTE LAA networks, a co-located WiFi network will be impacted drastically as it has to compete against more networks that are able to gain access to the channel for considerably longer duration.

In order to deal with this serious concern, we propose a new adaptive channel access scheme for LTE-U. According to this scheme, LTE has to perform a CCA before a transmission. If the CCA estimates the channel as idle, then the LTE LAA eNB transmits for a variable duration called TXOP in a range of 2 up to $20 \mathrm{~ms}$. This TXOP is followed by a variable muting period in a range of 0 up to $20 \mathrm{~ms}$. During the muting period, the LTE-U network that has finished a transmission of a TXOP duration has to remain silent in order to give channel access opportunities to other co-located networks (e.g. Wi-Fi or another LTE-U). After the end of the muting period (or at the end of the TXOP in case of zero muting period), the eNB has to perform again a CCA procedure before a new TXOP. In this solution, the introduction of the muting period can cause problems for delay sensitive traffic. In this case, similar to LTE LAA, a primary cell operating in licensed spectrum can still be used for time sensitive transmissions. In the rest of the article, we will refer to the proposed scheme as muting LTE-U (mLTE-U). Figure 3 illustrates the proposed scheme.

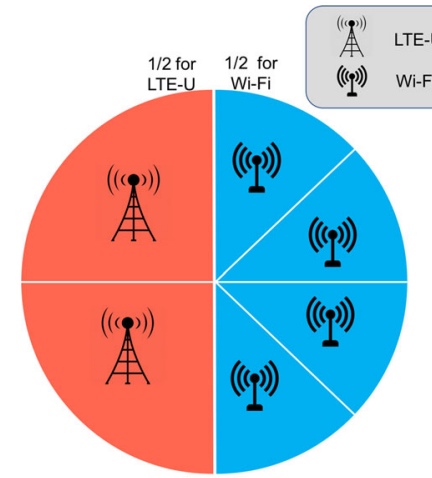

(a)

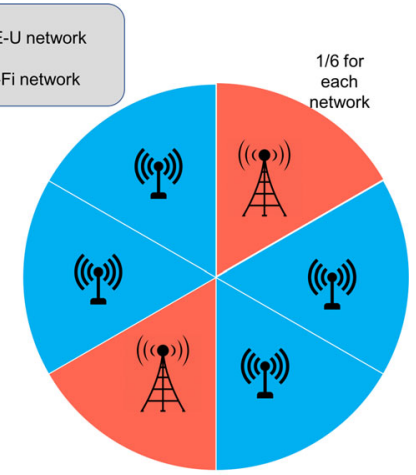

(b)
Fig. 4 Spectrum sharing between different co-located technologies. a spectrum sharing based on the different technologies b Spectrum sharing in technology-agnostic way

This scheme can offer high coexistence flexibility as the mLTE-U behaviour can be adapted based on various parameters, such as the number and the type of the co-located networks, the QoS requirements that a network has to serve (e.g. best effort traffic, video traffic, etc.) and the load of the different networks. For instance, when an mLTE-U network coexists with multiple Wi-Fi networks, then the proposed scheme has to be adapted so that mLTE-U transmits using a short TXOP followed by a relatively long muting period. The Wi-Fi networks can exploit this period to further gain channel access. According to another example-scenario, an mLTE-U that serves a video streaming coexists with a $\mathrm{Wi}-\mathrm{Fi}$ network that serves best-effort traffic. In this case, the mLTE$\mathrm{U}$ transmission scheme has to be modified in order to use a higher TXOP followed by a shorter muting period for $\mathrm{Wi}-\mathrm{Fi}$ transmissions.

\section{Fairness in unlicensed spectrum}

The purpose of the proposed scheme is to enhance the coexistence and increase the fairness among the co-located LTE-U and Wi-Fi networks. A fair coexistence scheme should offer all the available networks equal opportunities to the medium. It is important to point out the difference between fairness among different available technologies and fairness among the different coexisting networks, as it is depicted in Fig. 4.

According to the first approach (Fig. 4a), the wireless resources are divided among the co-located networks according to the different wireless technologies that are used. Hence, in the case of two coexisting wireless technologies such as LTE and Wi-Fi, half of the time the medium is used by LTE and half of the time is used by Wi-Fi. In our opinion, such an approach is not always fair as it does not take into consideration the number of the LTE and Wi-Fi networks respectively. For instance, if there are multiple co-located Wi-Fi networks 
and one LTE-U network, it would not be fair to Wi-Fi to split the time that the different technologies access the channel to the half.

Regarding the second approach (Fig. 4b), the medium is shared according to the number of the co-located networks in a technology-agnostic manner. Consequently, a coexistence mechanism that belongs in this category does not discriminate the coexisting networks based on the type of the wireless technology that they use. Instead, the distribution of the resources is done based on the number of the co-located networks and ideally based on several characteristics, such as the type and the amount of traffic that must be served.

In an ideal scenario in which all the different networks are aware of the requirements of each other and can exchange information, or a central coordinator is in charge of communicating with each network, collecting their traffic requirements and coordinating their transmissions, the distribution of the wireless resources could be done in a really fair manner.

However, in the wireless world, several diverse networks that have been designed, each having completely different principles in order to serve different requirements, are forced to coexist and compete for the wireless resources. Furthermore, the channel access mechanisms used by different technologies vary significantly among each other. Even between nodes of the same wireless technology equally time sharing of the wireless resources is not guaranteed. Wi-Fi is an indicative example of such a scenario. One of the basic principles of traditional Wi-Fi (without frame aggregation) is the equal division of the channel between the users. Hence, only one packet is transmitted by each node after the medium is sensed as idle. Nevertheless, very often there is a case in which a node faces better channel quality than another. Thus, the node with better channel conditions can perform a faster transmission, using a high Modulation and Coding Scheme (MCS) profile compared to the other. This way the node with the lower MCS profile occupies the channel for longer duration to transmit exactly the same number of bytes.

In the case of LTE and Wi-Fi coexistence, the two technologies that compete for the wireless resources are diverse having major design differences. The obtained throughput together with the channel occupancy are good indicators for the fairness that a coexistence technique can offer. Hence, in the rest of the article, the obtained throughput and the channel occupancy are adopted as key performance indicators for the evaluation of the proposed scheme. Towards a fair coexistence in line with the second approach that discussed above, the parameters of mLTE-U are selected in such a way that each participating network can achieve an equal ratio of throughput, compared to the maximum throughput that it can be achieved during the standalone operation.
Table 2 mLTE-U simulation parameters

\begin{tabular}{ll}
\hline Parameter & Value \\
\hline Base station type & Femtocell \\
Bandwidth & $20 \mathrm{MHz}$ \\
Defer period & $34 \mu \mathrm{s}$ \\
Slot duration & $9 \mu \mathrm{s}$ \\
$\mathrm{CW}_{\text {min }}$ & 15 \\
$\mathrm{CW}_{\text {max }}$ & 1023 \\
TXOP & $2-20 \mathrm{~ms}$ \\
Muting period & $0-20 \mathrm{~ms}$ \\
ED threshold & $-62.0 \mathrm{dBm}$ \\
CW update rule & $80 \% \mathrm{NACKS}$ \\
MIMO format & MIMO \\
\hline
\end{tabular}

\section{Simulation environment}

In order to evaluate the proposed scheme, experiments have been performed using the NS3 network simulator, which is an event-based and flexible simulation platform. The simulator allows the design of scenarios in which multiple LTE networks can coexist together with multiple Wi-Fi networks in the unlicensed spectrum. The LTE and Wi-Fi networks are able to operate using the same channel and can interfere with each other.

During the experiments, the LTE has been set to operate in the $5 \mathrm{GHz}$ unlicensed band. As it is mentioned in Sect. 4, mLTE-U can transmit using a variable TXOP period, which ranges from 2 up to $20 \mathrm{~ms}$. In addition, a muting period has been introduced to the LTE channel access scheme. This muting period ranges from 0 up to $20 \mathrm{~ms}$ and starts after the completion of a TXOP period. The maximum duration of both TXOP and muting period can be set to even higher values. However, we believe that this range is long enough to showcase the effect that the proposed scheme can have on the coexistence between mLTE-U and Wi-Fi networks in unlicensed spectrum.

Before an mLTE-U node starts a transmission, it has to complete a CCA procedure. The CCA parameters have been configured in order to be similar to the Wi-Fi LBT Category 4 procedure. Table 2 summarizes the specific mLTE-U parameters that have been used.

Regarding the Wi-Fi network, 802.11n mode has been selected in order to allow operation in $5 \mathrm{GHz}$ unlicensed band. Additionally, frame aggregation is disabled so that we can investigate the traditional 802.11 transmission, according to which a single packet is transmitted after the channel is estimated as idle. Additionally, the network is configured to operate in SISO mode, so that the Wi-Fi operation can be comparable to other popular 802.11 standards that does not 
Table 3 Wi-Fi simulation parameters

\begin{tabular}{ll}
\hline Parameter & Value \\
\hline Wi-Fi mode & $802.11 \mathrm{n}$ \\
Frame aggregation & Disabled \\
Bandwidth & $20 \mathrm{MHz}$ \\
DIFS duration & $34 \mu \mathrm{s}$ \\
Slot duration & $9 \mu \mathrm{s}$ \\
CW $_{\text {min }}$ & 15 \\
CW $_{\text {max }}$ & 1023 \\
ED threshold & $-62.0 \mathrm{dBm}$ \\
CS threshold & $-82.0 \mathrm{dBm}$ \\
RTS/CTS & Disabled \\
MIMO format & SISO \\
\hline
\end{tabular}

Table 4 Common simulation parameters

\begin{tabular}{ll}
\hline Parameter & Value \\
\hline Simulation time per mLTE-U configuration & $10 \mathrm{~s}$ \\
Traffic direction & Downlink \\
Traffic protocol & UDP \\
UDP payload size & 1472 bytes \\
Radio propagation model & Log-distance path loss \\
Antenna pattern & Omni-directional \\
TX power (eNB, AP) & $18 \mathrm{dBm}$ \\
TX power (UE, STA) & $18 \mathrm{dBm}$ \\
\hline
\end{tabular}

support MIMO mode such as $802.11 \mathrm{a} / \mathrm{g}$. Table 3 lists all the related parameters that have been used for the configuration of the Wi-Fi network.

The common simulator parameters are presented in Table 4 .

Before the beginning of a transmission burst mLTE-U must perform a CCA procedure. This means that the medium can be sensed as idle at any time. On the other hand, LTE is a scheduled technology and the scheduling is performed by the eNB on a sub-frame level, meaning that each $1 \mathrm{~ms}$ the assignment of the wireless resources to the active UE can change. Hence, as every data transfer starts at the subframe boundaries, an LTE reservation signal is used after the channel is sensed as idle and until the beginning of the next subframe in order to preserve the channel and force other nodes to backoff. In the best-case but very rare scenario in which the channel is estimated as idle in the beginning of a subframe, the transmission of a reservation signal is not necessary and thus it is omitted. Contrariwise, when the channel is sensed idle immediately after the beginning of a subframe, then the reservation signal lasts for the rest of the subframe and the data transmission starts at the beginning of the next

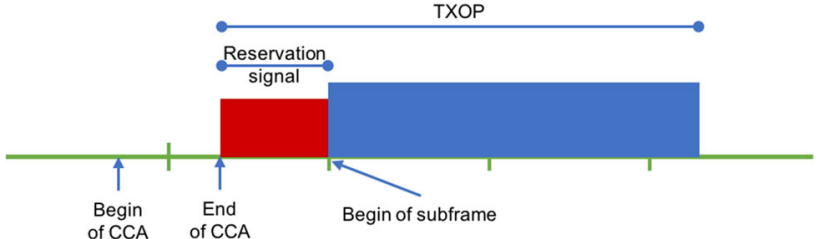

Fig. 5 mLTE-U reservation signal

subframe. The duration of the reservation signal is deducted from the TXOP duration of the mLTE-U. For this reason, the minimum examined TXOP is $2 \mathrm{~ms}$. Figure 5 illustrates the usage of the reservation signal as described above.

\section{Simulation scenarios}

In order to verify the coexistence issue that occurs when LTE LAA operates next to $\mathrm{Wi}-\mathrm{Fi}$, a related simulation scenario has been designed. According to this scenario, an LTE LAA network consisting of one eNB and one UE operates in the proximity of a Wi-Fi network that consist of one AP and one STA.

Towards the performance evaluation of the proposed scheme, various simulation scenarios have been designed. For each scenario, all the different combinations of TXOP and muting values have been tested. For both mLTE-U and Wi-Fi networks, we assume that one end-device is connected to one base station. In each network, high load UDP traffic is transmitted in the DL, meaning from the eNB to the UE for LTE and from the AP to the STA for Wi-Fi.

For the evaluation of the proposed scheme during the first four scenarios, the mobility of the end-nodes is not taken into consideration. In these scenarios, we study the performance of the mLTE-U scheme in cases of different mLTE-U and WiFi network densities. The first examined scenario consists of one mLTE-U network and one Wi-Fi network. The distance between the LTE eNB and the Wi-Fi AP is $10 \mathrm{~m}$, while the LTE UE and the Wi-Fi STA are located at a distance of $10 \mathrm{~m}$ from the eNB and the AP respectively. In the remainder of the article we refer to this scenario as reference scenario. For the other investigated static scenarios, the number of the mLTE$\mathrm{U}$ and Wi-Fi networks ranges from one up to four networks for each type of technology. This way, various situations of high interest can be studied, such as:

- Coexistence of low mLTE-U and Wi-Fi density (e.g. reference scenario)

- Coexistence of high mLTE-U density and low Wi-Fi density (e.g. 4 mLTE-U and $1 \mathrm{Wi}-\mathrm{Fi}$ )

- Coexistence of low mLTE-U density and high Wi-Fi density (e.g. $1 \mathrm{mLTE}-\mathrm{U}$ and $4 \mathrm{Wi}-\mathrm{Fi}$ ) 


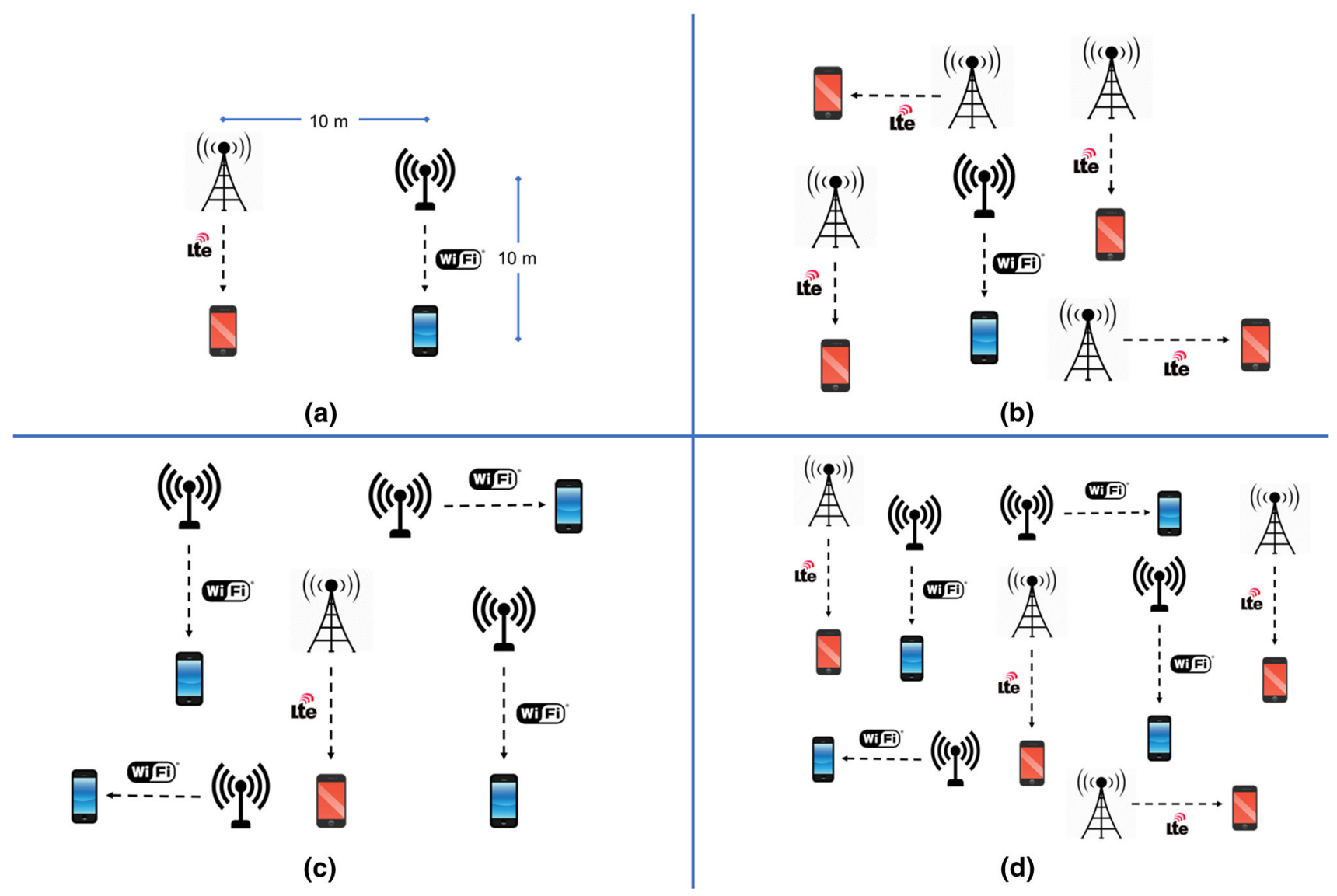

Fig. 6 Investigated static coexistence scenarios. a Reference scenario, b dense mLTE-U deployment scenario, c dense Wi-Fi deployment scenario, d dense mLTE-U and Wi-Fi deployment scenario

- Coexistence of both high mLTE-U and Wi-Fi density (e.g. 4 mLTE-U and 4 Wi-Fi)

In every scenario with multiple mLTE-U and/or multiple Wi-Fi networks, all the available nodes (eNBs, UEs, APs and STAs) are deployed randomly in the proximity of each other (within $20 \mathrm{~m}$ ). This way, the ED threshold is surpassed and the backoff mechanism of mLTE-U and Wi-Fi is triggered during every transmission. Figure 6 presents the investigated static coexistence scenarios.

Furthermore, the effect of mobility in the coexistence of mLTE- $\mathrm{U}$ and Wi-Fi is studied in an indicative mobile scenario. During the mobile scenario and similar to the reference scenario, one mLTE-U network, consisting of one eNB and one UE, coexists with one Wi-Fi network consisting of one $\mathrm{AP}$ and one STA. The UE is placed at a distance of 25 meters from the eNB and the STA is placed at a distance of 100 meters from the AP. During the execution of the scenario, the UE moves away from the eNB, while the STA moves towards the AP. The above described scenario is depicted in Fig. 7.

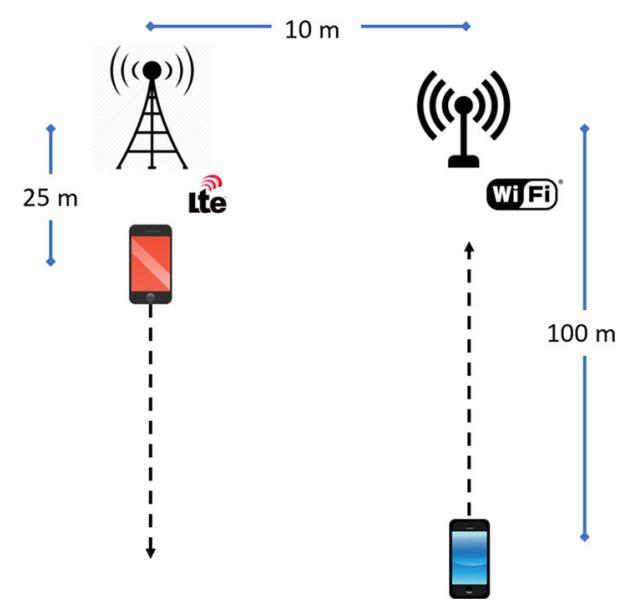

Fig. 7 Mobile coexistence scenario

\section{Performance evaluation}

\subsection{LTE LAA and Wi-Fi coexistence evaluation}

In Sect. 4, we discussed the coexistence problems that can arise when an LTE LAA network operates next to a Wi-Fi 
Table 5 Notations of the LTE LAA and Wi-Fi CCA procedure

\begin{tabular}{ll}
\hline Parameter & Meaning \\
\hline$N$ & Backoff counter \\
$T_{d}$ & The defer duration \\
$T_{f}$ & The silent period in the beginning of $T_{d}$ \\
$m_{p}$ & Number of backoff slots \\
$T_{s l}$ & Backoff slot duration \\
$T_{m c o t, p}$ & LTE transmission duration \\
$T_{F r a m e}$ & Wi-Fi frame transmission duration \\
SIFS & 802.11 Short Interframe Space \\
DIFS & 802.11 DCF Interframe Space \\
\hline
\end{tabular}

network in the unlicensed spectrum. This section, evaluates the impact of the different LTE LAA priority classes (Table 1) on the performance of Wi-Fi.

Figure 8 showcases the CCA procedure of LTE LAA that is configured to use priority class 3 . The upper part of the figure shows the CCA procedure, when LTE LAA is the only network in the unlicensed channel, while the lower part shows the procedure when LTE LAA coexists with a Wi-Fi network. The notations of the LTE LAA CCA procedure are specified in Table 5.

According to the LTE LAA standard, an LTE LAA transmission is performed, after the channel is sensed as idle during all the slots of a defer period $\left(T_{d}\right)$ and after the backoff counter $(N)$ is reached zero. A defer period consists of a silent period ( $T_{f}$ ), followed by $m_{p}$ slots of $T_{s l}$ duration. The number of slots $\left(m_{p}\right)$ is defined by the priority classes. An LTE LAA node that wants to transmit, first senses the medium for a defer period $\left(T_{d}=T_{f}+m_{p} * T_{s l}\right)$ and then, it always performs an exponential backoff. The backoff counter $N$ is chosen randomly in a range of $0 \leq N \leq C W$. In the beginning, the $C W$ is initialized to the $C W_{\min , p}$ value specified by the corresponding priority class. If during the backoff procedure the channel is sensed as busy, then the backoff counter freezes. The channel is reported as busy, when the sensed energy during a CCA slot is above the ED threshold. Every time the channel is sensed as busy, the LTE LAA node has to sense again for an idle defer period $\left(T_{d}\right)$ and then it continues decreasing the backoff counter from the point it stopped. This is depicted in the second half of the Fig. 8, where a Wi-Fi transmission occurs during the backoff procedure of LAA.

The value of the $C W$ is adjusted based on the HARQ feedback from the UEs. If the feedback indicates that at least $80 \%$ of the HARQ-ACK values, corresponding to the most resent DL transmission burst were erroneous (negative acknowledgments, NACKS), for example when a lot of collisions occur, then the $C W$ is doubled for the next CCA procedure. This can happen until the $C W$ reaches a maximum value $C W_{\max , p}$ specified by each priority class. If less than $80 \%$ of the HARQ-ACK values are determined as NACK, the $C W$ is reset to the minimum value.

In order to assess the coexistence offered by LTE LAA to a co-located Wi-Fi network, all the four priority classes have been tested via the simulation platform. The simulation platform has been modified in order to enable LTE LAA simulation. To this end, the defer period, the $\mathrm{CW}_{\min }$, the $\mathrm{CW}_{\max }$ and the TXOP have been adjusted to the corresponding values specified by each priority class. The Wi-Fi simulation parameters are the same as listed in Table 3. For all the four priority classes, the distance between the eNB and the AP is 10 meters and the distance between each base station with its respective end-device is 10 meters. High UDP traffic of $200 \mathrm{Mbps}$ is transmitted on the DL in both networks. Figure 9 presents the obtained throughput for LTE LAA and Wi-Fi (vertical axis) according to the corresponding LTE LAA priority class (horizontal axis). The Wi-Fi throughput in standalone operation (without LTE LAA interference) is also presented as a reference point.

From the graph, it can be seen that LTE LAA has a big impact on the performance of Wi-Fi. For the two higher priority classes, LTE LAA has priority over Wi-Fi due to the differences in the configuration of the channel estimation procedure that the two networks use. Wi-Fi uses always a DIFS period of $34 \mu \mathrm{s}$, while the $\mathrm{CW}_{\min }$ and $\mathrm{CW}_{\max }$ values are 15 and 1023 respectively. On the other hand, LTE LAA uses a short defer period $\left(\mathrm{T}_{\mathrm{d}}\right)$ and shorter ranges for the selection of the CW (Table 1). Regarding the two lower priority classes the configurations of the channel estimation procedure are more in line with the Wi-Fi LBT procedure. Nevertheless, LTE LAA uses transmission bursts $\left(T_{m c o t}, p\right)$ of significant longer duration ( 8 or $10 \mathrm{~ms}$ ) compared to a typical Wi-Fi packet transmission that lasts some hundreds of $\mu \mathrm{s}$. Hence, Wi-Fi is able to achieve a maximum throughput of 3.32 Mbps when LTE LAA is configured with the lowest priority class and uses the shorter possible transmission burst ( 8 $\mathrm{ms})$. However, the obtained throughput is significantly lower compared to the throughput that Wi-Fi can achieve in a standalone operation (30.44 Mbps).

The graph also showcases that the different priority classes have an effect on the LTE LAA throughput. According to the two higher priority classes, LTE LAA transmits for 2 and $3 \mathrm{~ms}$ respectively before it estimates the channel again. On the contrary, according to the two lower priority classes, LTE LAA transmits for 8 or $10 \mathrm{~ms}$ after a successful CCA procedure. This means that for the higher classes it performs a CCA procedure more often than for the lower. As a result, it spends more time assessing the channel and this has an immediate effect on the obtained throughput.

In every case, the simulation results show that LTE LAA can degrade the performance of Wi-Fi in its classic form, meaning that no frame aggregation is used and it transmits one packet every time the medium is sensed as idle. In the rest 


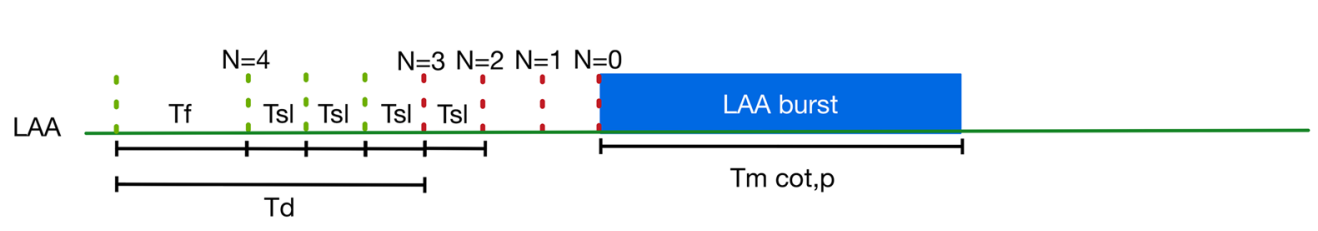

$\mathrm{N}=$ Ninit $=4$

$\mathrm{Td}=\mathrm{Tf}+\mathrm{mp} \mathrm{p}^{*} \mathrm{Ts}$

$\mathrm{mp}=3$

$\mathrm{Tf}=16$ us

Ts $=9$ us

$\mathrm{DIFS}=\mathrm{SIFS}+2^{\star} \mathrm{Ts}$

$\operatorname{SIFS}(5 \mathrm{GHz})=16$ us
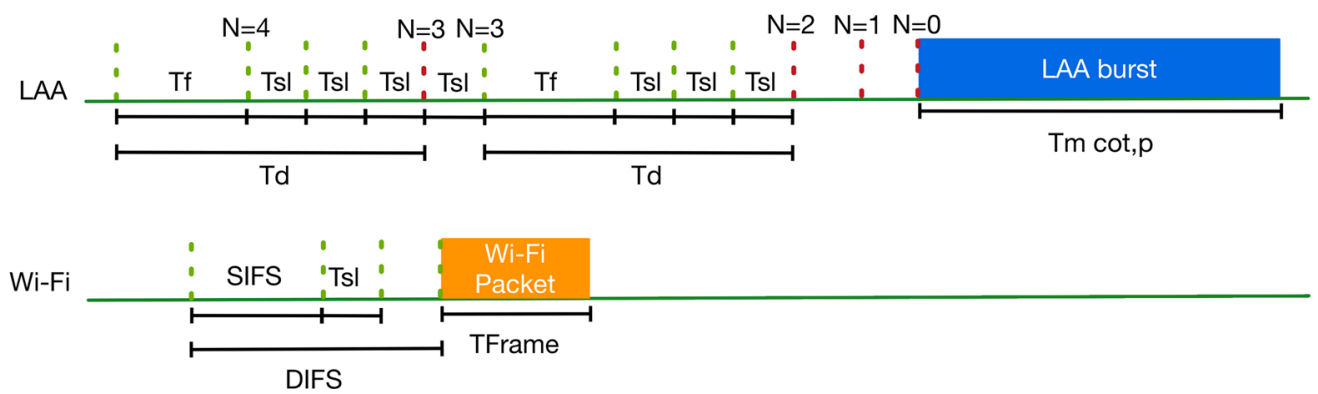

Fig. 8 LTE LAA CCA procedure

of this section, we evaluate the performance of the proposed mLTE-U scheme under different scenarios and we discuss what configurations of TXOP and muting period can offer fair coexistence for each scenario.

\subsection{Standalone scenario evaluation}

In this section, the performance of both mLTE-U and Wi-Fi networks is evaluated in standalone case. In this scenario, the networks are located away from each other and operate independently, having full access to the channel. The distance between the networks is set to $1000 \mathrm{~m}$ and both systems are offered an equal UDP load of $200 \mathrm{Mbps}$.

Figure 10 shows the obtained DL throughput results of mLTE-U network. On the $\mathrm{X}$-axis are the configurations of muting period duration in ms ranging from 0 up to 20 . On the $\mathrm{z}$-axis are the different TXOP configurations in ms ranging from 2 up to 20. Finally, on the y-axis are the DL throughput values in Mbps for every combination of TXOP and muting period durations. As it can be observed and according to the expectations, the introduction of the muting period has an impact on the maximum throughput that can be achieved by mLTE-U.

This graph shows clearly how the mLTE-U throughput drops as the TXOP period decreases and the muting period increases. Hence the minimum throughput value corresponds to a configuration in which TXOP lasts for $2 \mathrm{~ms}$ and is followed by a muting period that lasts $20 \mathrm{~ms}$. Respectively, the maximum throughput value corresponds to a TXOP of $20 \mathrm{~ms}$ followed by a muting period of $0 \mathrm{~ms}$. The difference between the maximum and the minimum value of the DL throughput reaches $95.2 \%$.

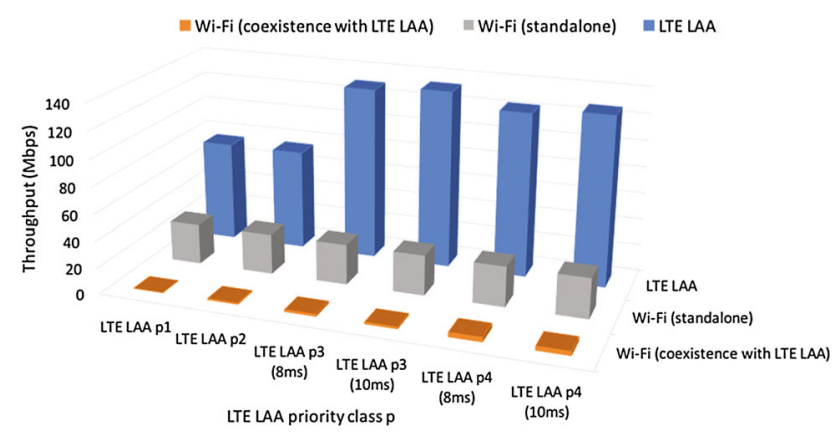

Fig. 9 LTE LAA and impact on Wi-Fi throughput for different channel access priority classes

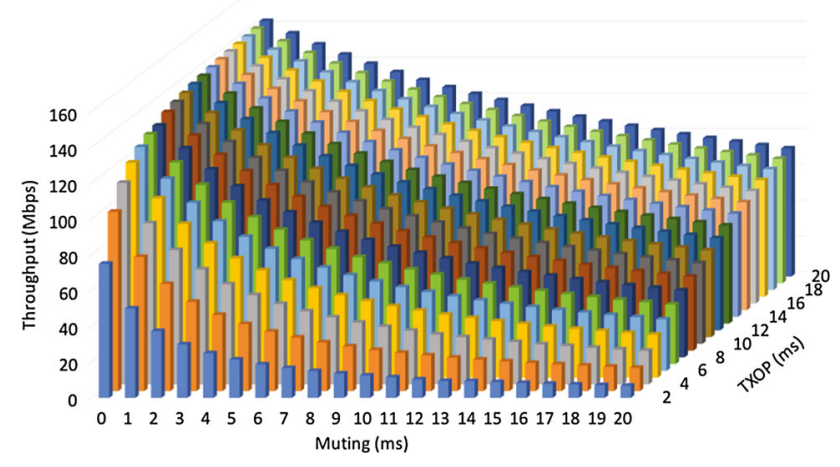

Fig. 10 Obtained mLTE-U throughput during the standalone scenario

Figure 11 shows the DL throughput diagrams of mLTE-U and $\mathrm{Wi}-\mathrm{Fi}$, when the mLTE-U muting period is zero and the mLTE-U TXOP period varies from 2 to $20 \mathrm{~ms}$. When the muting period is zero the mLTE-U can reach the maximum throughput for each corresponding TXOP.

As can be seen from the graph, the Wi-Fi throughput remains constant at $30.44 \mathrm{Mbps}$. This is to be expected as the 


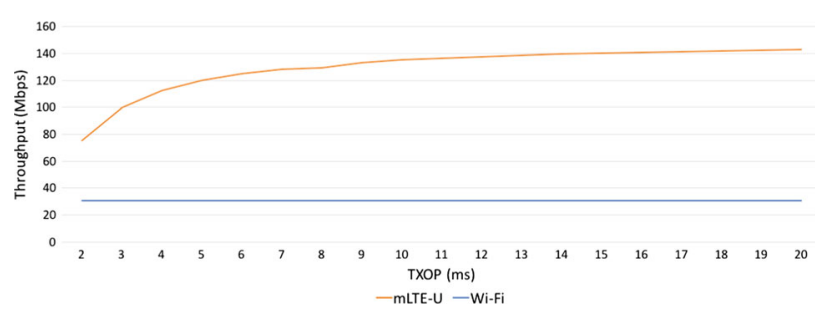

Fig. 11 Standalone mLTE-U and Wi-Fi throughput for different TXOP without muting period

Wi-Fi network is not in the proximity of mLTE-U and thus it is not affected by its transmissions. On the contrary, the mLTE-U throughput ranges from 75.18 up to $142.81 \mathrm{Mbps}$. This variation is related to the configured TXOP duration of the mLTE-U. As the TXOP duration decreases, the mLTE-U has to perform more often a CCA procedure in order to evaluate the status of the channel. This has a significant impact on channel utilization and respectively on the obtained throughput. For a lower TXOP duration the eNB spends more time evaluating the channel compared to the scenario in which it is configured with a higher TXOP duration.

This becomes clearer by comparing two different mLTE-U configurations for the standalone scenario. For both configurations, DL traffic is transmitted for $10 \mathrm{~s}$. According to the first configuration, the eNB transmits for a TXOP of $20 \mathrm{~ms}$ and each TXOP is followed by a muting period of $20 \mathrm{~ms}$. Hence, it evaluates the channel every $40 \mathrm{~ms}$. This means that the total number of CCA performed during the whole experiment is $10000 / 40 \mathrm{~ms}=250$ channel evaluations. When the eNB transmits for a TXOP of $4 \mathrm{~ms}$ followed by a muting period of $4 \mathrm{~ms}$, then the channel is sensed every $8 \mathrm{~ms}$. This corresponds to $10000 / 8 \mathrm{~ms}=1250$ channel evaluations.

Another parameter of high interest that is closely related to the obtained throughput is the channel occupancy time. The obtained simulation results show that during the standalone operation, Wi-Fi occupies the channel for $70.10 \%$ of the time. This means that Wi-Fi spends a high percentage of time sensing the medium. On the other hand, in the proposed scheme mLTE-U achieves the highest channel occupancy when the muting period is configured to be zero. In that case, mLTE-U competes for the medium immediately after the end of the TXOP. Figure 12 shows the percentage of channel occupancy for both Wi-Fi and mLTE-U for every TXOP duration and for muting period equal to zero.

As it has been discussed above, the TXOP duration is closely related to the frequency of CCA procedure. As it is illustrated in Fig. 12, for the lower values of TXOP the CCA frequency has a small impact on the channel occupancy of CCA. It must be noted that the transmission of the mLTE$\mathrm{U}$ reservation signal is counted in the computation of the channel occupancy. However, the transmission of the reservation signal is not taken into account for the computation

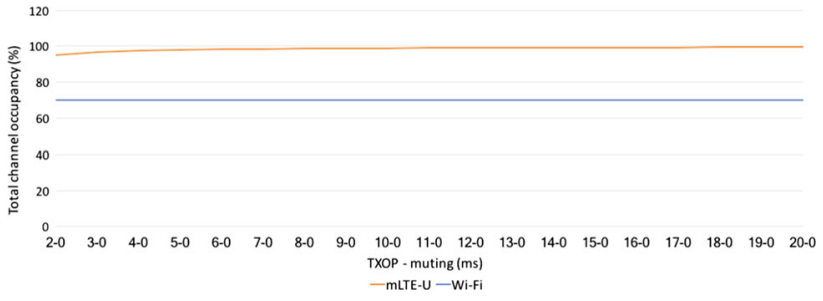

Fig. 12 Channel occupancy of mLTE-U and Wi-Fi during the standalone scenario

of the obtained throughput. For this reason, the throughput drop that can be observed in Fig. 11 for lower TXOP is not reflected in the achieved mLTE-U channel occupancy that is depicted in Fig. 12. Furthermore, this figure showcases the high spectral efficiency of LTE, especially in a clear environment. LTE can achieve high spectral efficiency as it is a scheduled technology that uses a centralized MAC protocol and was originally designed to operate in the licensed spectrum. During this standalone scenario, the percentage of the mLTE-U channel occupation ranges from $94.90 \%$ for $2 \mathrm{~ms}$ of TXOP duration up to $99.47 \%$ for 20 ms of TXOP duration. The addition of the muting period following a TXOP can provide fairness among mLTE- $\mathrm{U}$ and $\mathrm{Wi}-\mathrm{Fi}$ at the cost of decreasing the spectral efficiency and the throughput of mLTE-U. However, towards a fair coexistence between different technologies concessions must be made. Moreover, mLTE-U can use the licensed anchor to accomplish critical transmissions, such as the control signals or serve applications with high QoS requirements.

\subsection{Reference scenario evaluation}

The reference scenario is similar to the standalone scenario with the difference that the two networks are placed in the proximity of each other. Figure 6 a illustrates the reference scenario. In this scenario, the two networks have to compete for the medium before a transmission.

Figure 13 shows the obtained DL throughput of the WiFi network. The x-axis is the TXOP duration of mLTE-U in $\mathrm{ms}$ and the z-axis is the muting period of mLTE-U in ms. The y-axis shows the DL Wi-Fi throughput for each different combination of mLTE-U TXOP and muting period. Figure 14 presents the DL throughput of the mLTE-U network. In this diagram, the $\mathrm{x}$ and $\mathrm{z}$ axes are reversed compared to Wi-Fi. Hence the $\mathrm{x}$-axis holds the muting period and the $\mathrm{z}$-axis holds the TXOP duration of mLTE-U. By observing the diagrams, it can be seen that they are inverse of each other. In case of $\mathrm{Wi}-\mathrm{Fi}$, the throughput increases as the muting period of mLTE-U increases. This is logical as highest mLTE-U muting period offers more opportunities to Wi-Fi to estimate the channel as idle and start a transmission. Furthermore, the WiFi throughput is inversely proportional to the mLTE-U TXOP. 


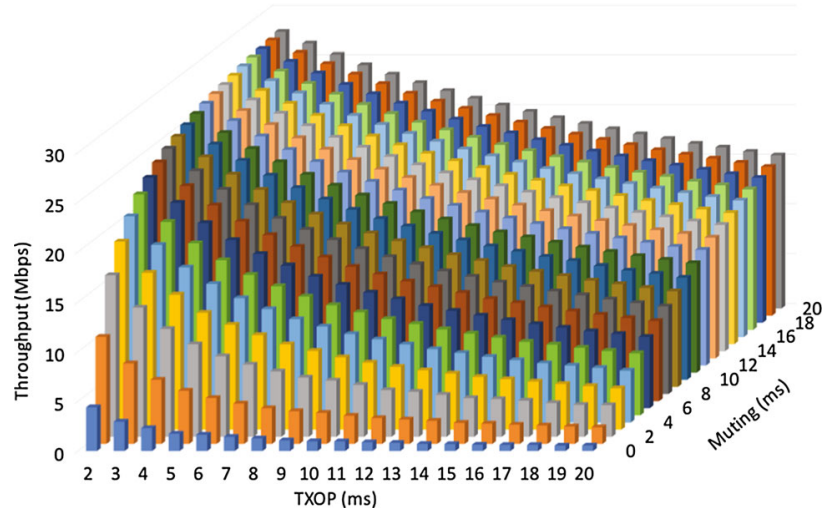

Fig. 13 Wi-Fi throughput during the reference scenario

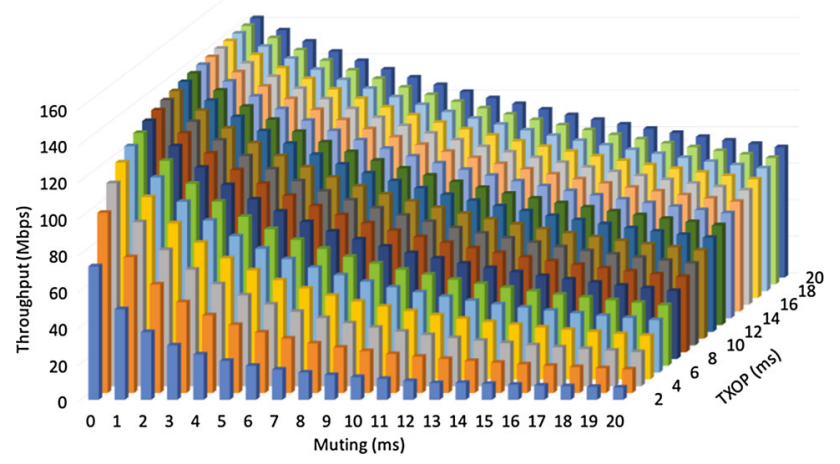

Fig. 14 mLTE-U throughput during the reference scenario

As it is explained above, a shorter TXOP gives more often opportunities to Wi-Fi to compete for the medium and eventually gain access to the channel. On the contrary, similar to the standalone scenario the throughput of mLTE-U increases when the TXOP duration increases due to less often CCA procedure. Additionally, as it is expected, a shorter muting period offers higher throughput compared to a longer one.

Comparing the reference scenario with the standalone operation, it can be observed that during the reference scenario, the mLTE-U throughput is slightly lower (less than 2 Mbps of throughput drop). This is justified by the fact that in this scenario, the two networks compete for the channel access. As result, Wi-Fi can win several CCA battles. A Wi-Fi transmission typically lasts for few hundreds of $\mu$ s. Hence, the impact of the Wi-Fi network on mLTE-U due to the CCA procedure is not so significant. On the other hand, the presence of the mLTE-U has an impact on Wi-Fi throughput compared to the standalone scenario where it was constantly nearly to $30 \mathrm{Mbps}$. The results show that the $\mathrm{Wi}-\mathrm{Fi}$ throughput can drop to $0.56 \mathrm{Mbps}$ in case that the eNB uses a TXOP of $20 \mathrm{~ms}$ and a muting period of 0 . In this case mLTE$\mathrm{U}$ occupies the channel for long time and competes for the medium immediately after the end of the TXOP. Thus, WiFi transmits only when it wins the CCA battle. Accordingly,

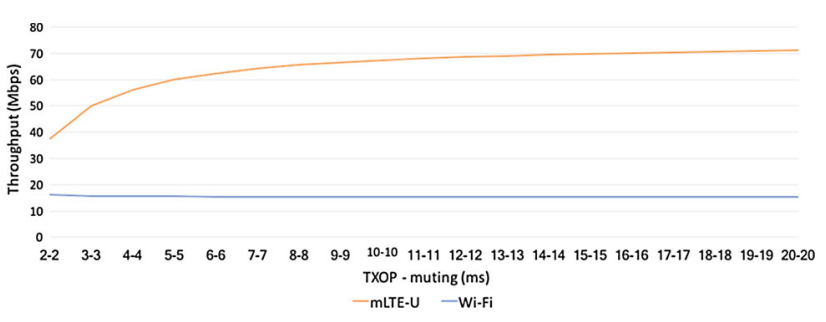

Fig. 15 mLTE-U and Wi-Fi fair throughput during the reference scenario

the Wi-Fi throughput reaches its peak, which is $27.80 \mathrm{Mbps}$, when the mLTE-U uses the longest muting period of $20 \mathrm{~ms}$ and the shortest TXOP of $2 \mathrm{~ms}$. Then mLTE-U remains silent the most of the time and gains access for short TXOP. The difference between the maximum and minimum throughput corresponds to $97.99 \%$.

As it has been discussed in Sect. 5, in order to share the channel in a fair manner, it must be ensured that all the co-located networks can gain equal opportunities to the medium. Regarding the reference scenario, it is expected that fair coexistence can be achieved when the mLTE-U network is configured with a TXOP and a muting period of the same duration. In Fig. 15 both the mLTE-U and the Wi-Fi throughput are depicted for every pair of TXOP and muting period of the same duration. Comparing this figure with Fig. 11, it can be observed that during the reference scenario, mLTE-U and Wi-Fi are able to achieve almost half of the throughput that could be reached during the standalone operation. Regarding the mLTE-U, for every pair of TXOP and muting period it achieves marginally lower throughput than the half of the standalone scenario. In the contrast, Wi-Fi obtains slightly higher throughput than the half that it can reach during the standalone operation. This is justified by the fact that the two networks compete for the channel access. mLTE-U transmits for a TXOP duration and then it remains silent for the same period of time. Wi-Fi can access the medium during this period that in a wider scale equals to the half duration of the experiment. Furthermore, Wi-Fi is possible to win multiple CCA battles. In this case, it can transmit a packet for each one of the idle channel assessments, gaining in total a slightly higher throughput than the half of the standalone operation. As result, mLTE-U throughput is limited marginally below the half of the standalone scenario.

The percentage of channel occupancy for both mLTE-U and Wi-Fi networks and for every pair of TXOP and muting period of the same duration are presented in Fig. 16. This graph points out the superiority of mLTE-U over WiFi regarding the spectral efficiency. As the TXOP duration increases, the channel occupancy of mLTE-U increases, approaching the highest possible value of $50 \%$. As the TXOP duration decreases, mLTE-U has to perform more often a CCA procedure. This decreases its spectral efficiency as more 


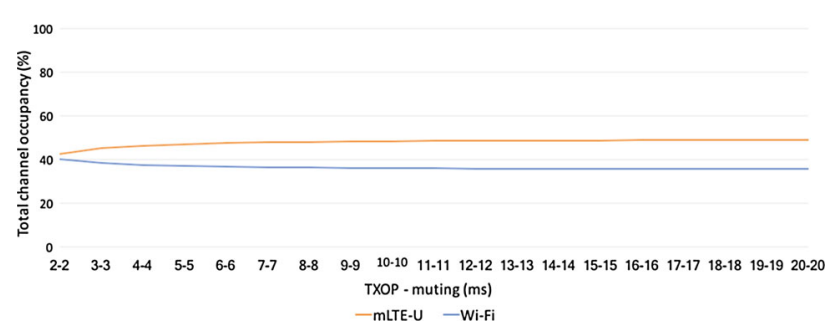

Fig. 16 Channel occupancy of mLTE-U and Wi-Fi towards fair coexistence during the reference scenario

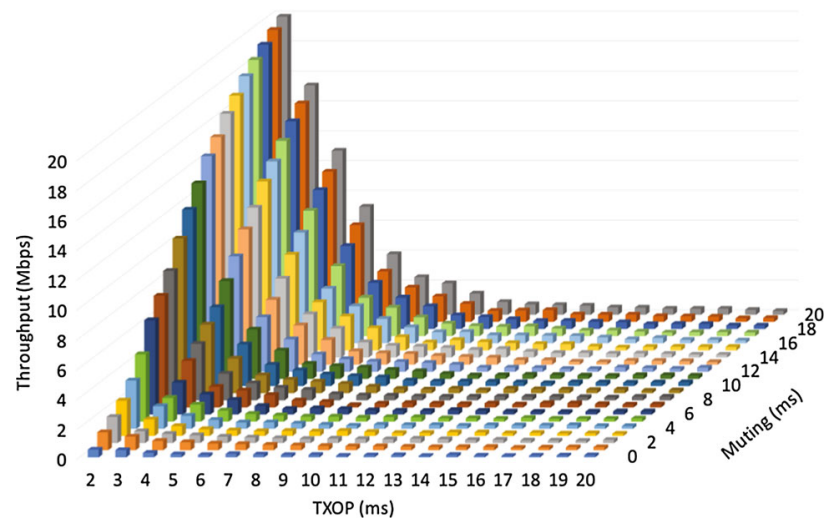

Fig. 17 Wi-Fi throughput during the dense mLTE-U scenario

time is spent in estimating the channel conditions. Regarding $\mathrm{Wi}-\mathrm{Fi}$, its channel occupancy slightly increases as the TXOP of mLTE-U decreases. This is again related to the frequency of mLTE-U channel estimation. A high CCA frequency (low TXOP) increases the probabilities of Wi-Fi to win the channel and transmit, increasing this way its total channel occupancy.

\subsection{Dense mLTE-U deployment scenario evaluation}

In this section, we evaluate the performance of the proposed scheme under a dense mLTE-U deployment scenario. In this scenario, one Wi-Fi and four mLTE-U networks operate in the proximity of each other. Figure $6 \mathrm{~b}$ illustrates the described scenario.

Figures 17 and 18 present the obtained throughput of the Wi-Fi network and the combined throughput of the mLTE-U networks respectively. In this scenario, the Wi-Fi throughput is highly affected by the presence of the four mLTE-U networks.

Under an mLTE-U dense deployment, the possibilities of a muting period to be exploited by another mLTE-U network are very high, especially when they are configured to use high TXOP duration and low muting period.

In the contrary, when the mLTE-U networks are configured to use a short TXOP and a high muting period, they remain silent simultaneously for a longer period of time. WiFi can exploit these periods in order to transmit. Hence, the

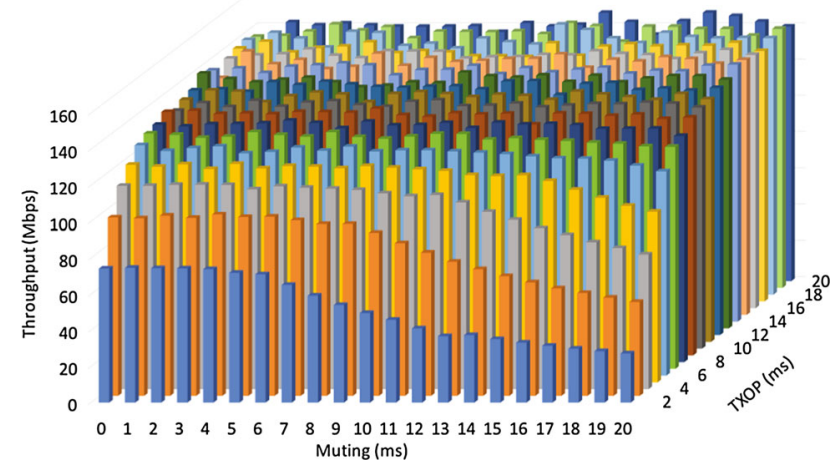

Fig. 18 mLTE-U combined throughput during the dense mLTE-U scenario

configuration of the highest muting period $(20 \mathrm{~ms})$ and the shortest TXOP $(2 \mathrm{~ms})$ offers the highest combined muting period (12 ms) for Wi-Fi. Then, Wi-Fi can achieve the highest throughput that corresponds to $19.96 \mathrm{Mbps}$. It worth to mention that this value is relatively higher than the achieved throughput during the reference scenario where the mLTE-U was configured with TXOP of $8 \mathrm{~ms}$ followed by a muting period of $12 \mathrm{~ms}$, which was $18.46 \mathrm{Mbps}$. This difference can be explained by the possibility of multiple mLTE-U transmissions to start simultaneously meaning that more than one $\mathrm{CW}$ counters reached zero at the same time. In this situation, the multiple mLTE-U transmissions will interfere with each other, giving the same time higher combined muting period to Wi-Fi.

By the time a TXOP starts, the transmitting node does not sense the medium for other concurrent transmissions. When multiple mLTE-U nodes start transmitting simultaneously, the interference caused by longer TXOPs has bigger impact compared to the shorter ones. This is the reason that the combined throughput graph of mLTE-U fluctuates during the longer TXOPs. This observation is also valid for LTE LAA operation and especially for lower priority classes that the duration of the transmission burst is longer.

Mechanisms that are able to deal with the interference between multiple mLTE-U transmissions are required. According to a possible solution, the transmitting node could periodically (e.g. every $2 \mathrm{~ms}$ ) pause its transmission in order to sense the medium for other potential transmissions for a short period of time (e.g. a defer period of $16 \mu$ s). If during this period the medium is idle, then it continues its transmission without performing a backoff. Otherwise, if the medium is busy, it can postpone its transmission and perform a CCA procedure. Techniques such as enhanced Inter-Cell Interference Coordination (eICIC) [29] that is designed to mitigate intrafrequency interference could be also part of the solution. Further study of interference management between different mLTE-U nodes is not in the scope of this article and is considered as future work. 


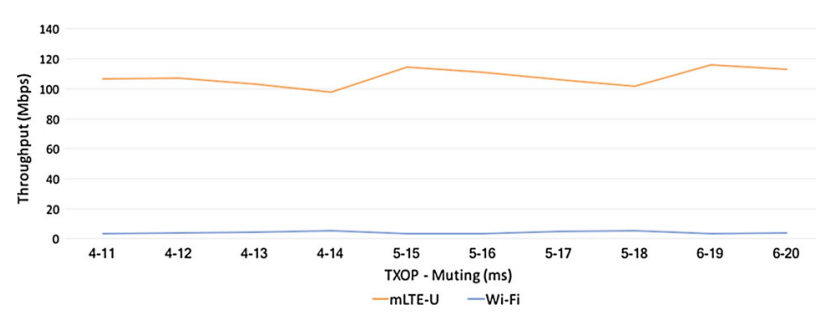

Fig. 19 mLTE-U configurations that can enable fair coexistence with Wi-Fi during the dense mLTE-U scenario

In order to achieve fair coexistence between the different co-located mLTE-U and Wi-Fi networks, the wireless resources must be equally distributed between them. When fairness is considered in terms of throughput, each one of the five networks must be able to obtain $20 \%$ of the throughput that can be achieved in the standalone scenario. This corresponds to 6.09 Mbps for Wi-Fi and to 28.56 Mbps for each one of the mLTE-U networks (114.24 Mbps combined mLTE-U throughput). Hence, from all the possible configurations of TXOP and muting period must be chosen the ones that provide a throughput that approaches these values. Figure 19 illustrates the Wi-Fi and the mLTE-U combined throughput for the configurations that can enable fair coexistence.

As can be observed, in this scenario mLTE-U can enable fair coexistence with Wi-Fi when it is configured with a relatively low TXOP followed by long muting period. This is something to be expected as from Fig. 17 is clear that for configurations of high TXOP followed by muting periods of varying duration, mLTE-U have a deep impact on Wi-Fi. As it has been discussed earlier, a long muting period in combination with a short TXOP offers to Wi-Fi more often a common muting slot, during which it can transmit.

\subsection{Dense Wi-Fi deployment scenario evaluation}

In this section, we study another scenario of high interest in which one mLTE-U network coexists with a dense Wi-Fi deployment consisting of four Wi-Fi networks. Figure $6 \mathrm{c}$ illustrates the examined topology. Figure 20 presents the combined throughput of Wi-Fi and Fig. 21 the obtained throughput of mLTE-U.

As it can be observed, during this scenario Wi-Fi can achieve a combined throughput similar to the reference scenario. The maximum combined Wi-Fi throughput approaches the 27.12 Mbps. This value is slightly lower than the respectively value of the reference scenario (27.80 Mbps), due to the multiple Wi-Fi networks that compete to access the shared channel. As it is expected, this value is achieved when mLTE-U is configured with the lowest TXOP followed by the highest muting period.

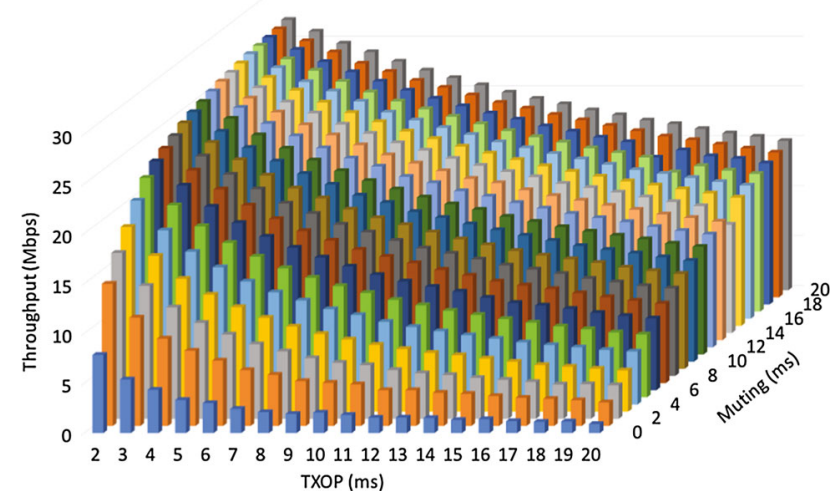

Fig. 20 Wi-Fi combined throughput during the dense Wi-Fi scenario

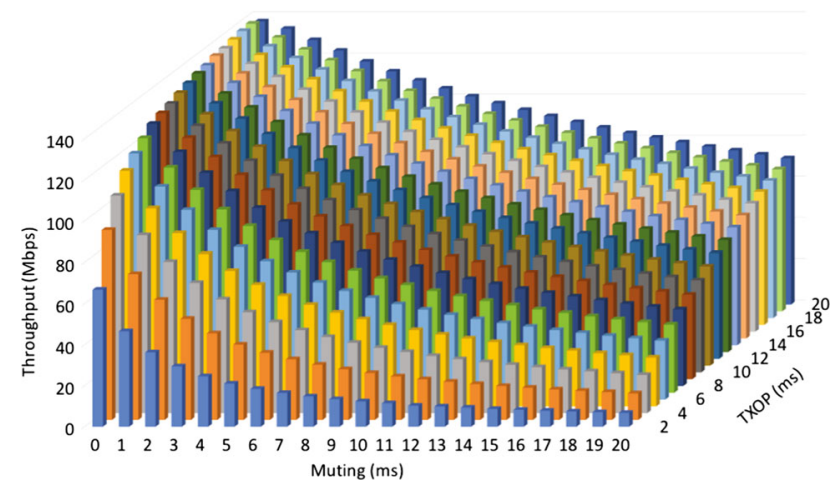

Fig. 21 mLTE-U throughput during the dense Wi-Fi scenario

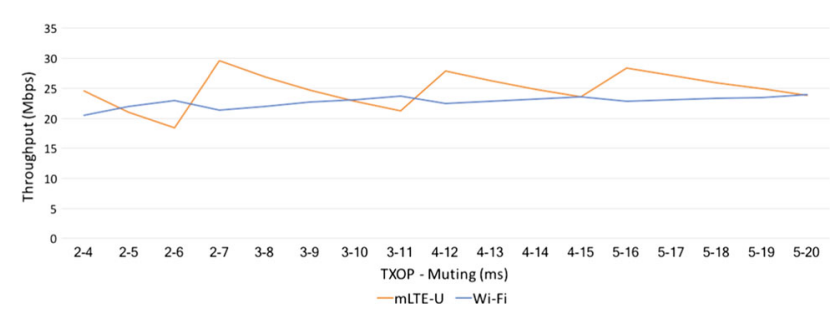

Fig. 22 mLTE-U configurations that can enable fair coexistence with Wi-Fi during the dense Wi-Fi scenario

Similar to the dense mLTE-U deployment scenario, fair coexistence can be achieved when all the co-located networks have equal opportunities to the wireless resources. Consequently, each one of the five networks must be able to achieve $20 \%$ of the throughput that can be reached during the corresponding standalone scenario. This equals to 6.09 Mbps for each Wi-Fi network (24.36 Mbps combined Wi-Fi throughput) and to $28.56 \mathrm{Mbps}$ for the mLTE-U network. Figure 22 depicts the TXOP and muting period configurations that offer fair coexistence in terms of equivalent throughput ratio among the different networks.

The graph reveals that fair coexistence can be attained when relatively low TXOP durations are used. The corresponding muting period can be configured in a wider range 


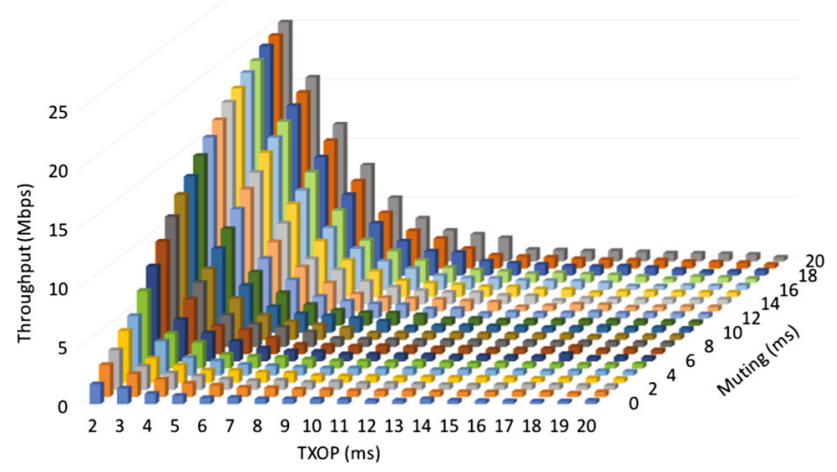

Fig. 23 Wi-Fi combined throughput during the dense mLTE-U and dense Wi-Fi scenario

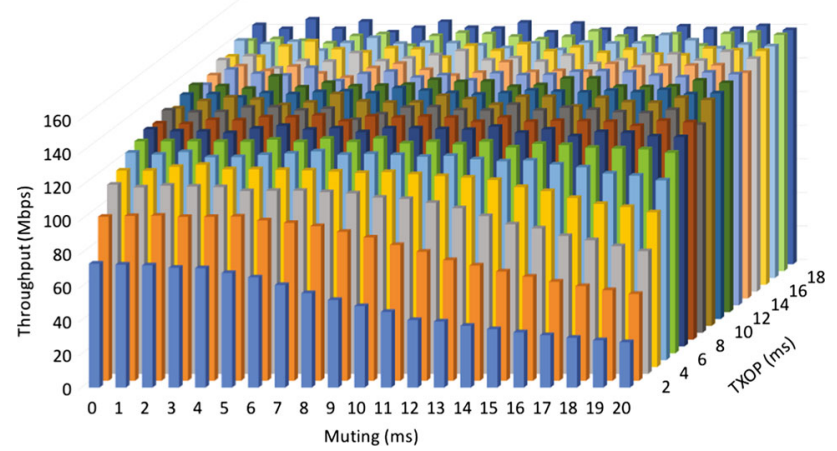

Fig. 24 mLTE-U combined throughput during the dense mLTE-U and dense Wi-Fi scenario

of values. As only one mLTE-U network coexists with multiple Wi-Fi networks, a short TXOP can offer more often CCA opportunities and a muting period during which the Wi-Fi networks can compete for the medium.

\subsection{Dense $\mathrm{mLTE}-\mathrm{U}$ and Wi-Fi deployment scenario evaluation}

This section showcases the performance of the proposed scheme under both dense mLTE-U and Wi-Fi deployment scenario. As it is presented in Fig. 6d, this scenario consists of four mLTE-U networks and four Wi-Fi networks. Each network is in the proximity of the others.

The combined throughput of Wi-Fi and of mLTE-U are shown in Figs. 23 and 24 respectively.

Figure 23 indicates that the Wi-Fi networks are clearly impacted by the coexisting mLTE-U networks in the majority of the configurations. However, when mLTE-U is configured with short TXOP and relatively long muting period durations the combined Wi-Fi throughput is significantly improved. The maximum combined throughput of Wi-Fi reaches 20.20 Mbps and it corresponds to $66.36 \%$ of the throughput that a Wi-Fi network can achieve during the standalone scenario.
100

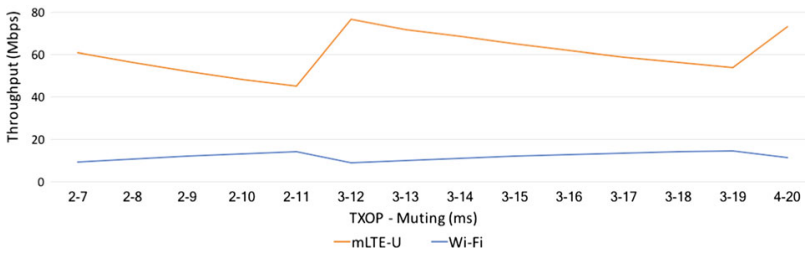

Fig. 25 mLTE-U configurations that can enable fair coexistence with Wi-Fi during the dense mLTE-U and Wi-Fi scenario

The multiple mLTE-U networks competing for the medium offer limited opportunities to Wi-Fi similarly to the scenario described in Sect. 8.4. In addition, due to the presence of multiple Wi-Fi networks the exploitation of these opportunities becomes even less optimal as they compete among each other to access the channel. On the other hand, the mLTE$\mathrm{U}$ networks achieve a maximum combined throughput that approaches the throughput that it can be reached in the standalone case.

Towards a fair coexistence, the selection of TXOP and muting period must be done in a way that all the co-located networks are able to reach the $1 / 8$ of the respective throughput of the standalone scenario. This means that each mLTE-U network must be able to achieve a maximum of $17.75 \mathrm{Mbps}$, while each Wi-Fi network must be able to reach around 3.75 Mbps. In terms of combined throughput mLTE-U should obtain 71.4 Mbps and Wi-Fi should be able to reach 15.2 Mbps. Figure 25 shows the TXOP and muting period values that can offer throughput that approaches the desired values for both mLTE-U and Wi-Fi.

As can be seen from the graph, fair coexistence can be achieved when the mLTE-U networks are configured with relatively low TXOP duration values. These TXOP values are followed by a muting period that varies from average to higher values as the TXOP increases. In this dense scenario, the selected values give short TXOP to mLTE-U followed by longer muting period during which the Wi-Fi networks compete and access the medium.

\section{7 mLTE-U and Wi-Fi mobile scenario evaluation}

In the previous described scenarios, all the end-devices (UE and STAs) were deployed statically, targeting to showcase the behavior of the proposed mLTE-U scheme in different density scenarios of high interest. This section discusses the effect of mobility when mLTE-U coexists with Wi-Fi. As illustrated in Fig. 7, one mLTE-U network coexists with one Wi-Fi network. The UE moves away from the eNB, while the STA moves towards the AP.

Figures 26 and 27 show the channel occupancy of mLTE$\mathrm{U}$ and Wi-Fi for the configurations of mLTE-U that can enable fair coexistence and for different distances of each 


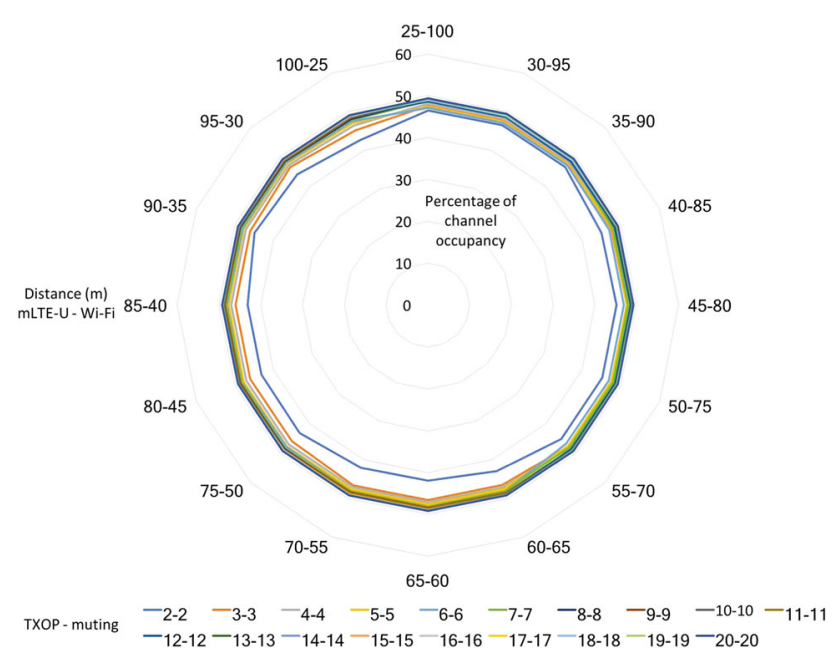

Fig. 26 mLTE-U fair channel occupancy for different distances between the UE and the eNB and between the STA and the AP

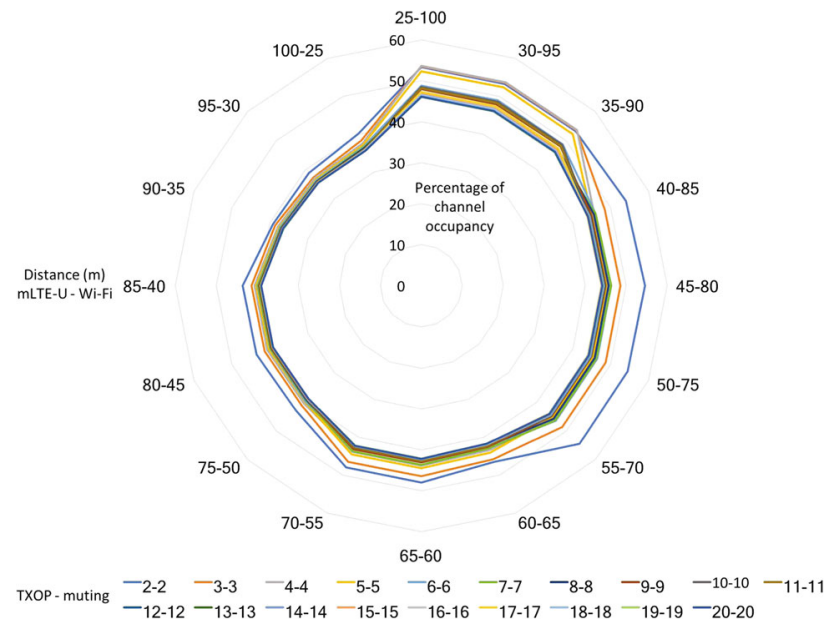

Fig. 27 Wi-Fi fair channel occupancy for different distances between the UE and the eNB and between the STA and the AP

end-device from the corresponding base station. The left part of the distance pairs represents the distance between the eNB and the UE, while the right part represents the distance between the AP and the STA. As can be seen from the graphs, the mLTE-U configurations that offer fairness are the same as the ones selected during the reference scenario (Sect. 8.3). These configurations correspond to the pairs, in which TXOP and muting period have equal duration.

In Fig. 26 can be seen that the channel occupancy of mLTE-U for the selected configurations ranges from 42.5 to $49.4 \%$. The difference in the percentage of channel occupancy lies in the fact that as the TXOP duration decreases, mLTE-U has to perform more often a CCA procedure spending more time in estimating the channel. Hence, when a longer TXOP duration is used, the spectral occupancy is increased approaching the highest possible value of $50 \%$ for the case of two coexisting networks. In Fig. 27 can be observed that the channel occupancy of Wi-Fi increases for longer distances between the AP and the STA and decreases for smaller distances. As an end-device moves far away from the associated base station, a lower MCS profile is used to render the wireless link more robust and able to cope with the decreased channel quality. However, a lower MCS profile corresponds to an increased channel occupancy, as a transmission requires more time compared to the case when a higher MCS profile is used.

As discussed in Sect. 5, fair coexistence refers to equal occupancy of the channel. Hence, for this definition of fairness and for the proposed coexistence scheme, the mobility of the end-devices does not affect the selection of the mLTE$\mathrm{U}$ configurations that can enable fair sharing of the spectral resources.

\section{Automatic fair parameter selection}

In the previous sections, the proposed scheme has been evaluated for different scenarios of high interest. Each of the scenarios investigates different density for mLTE-U and WiFi networks and identifies the combinations of TXOP and muting period that can provide fair coexistence between LTE and Wi-Fi. The fair coexistence is defined in terms of equal throughput ratio achievement for each one of the co-located networks. In the investigated scenarios, all the networks consist of one end-device connected to one base station and they have equal traffic requirements. As it has been revealed from the simulation results, for each scenario multiple configurations can provide the desired fair coexistence. The biggest challenge is to identify and select the optimal parameter values that can guarantee fair coexistence.

This section discusses how these configurations can be automatically identified. This identification can be done taking several parameters into consideration, such as the amount of the co-located networks and the type of traffic that must be served. The traffic that must be served refers to the load of each network and the QoS requirements. The degree that a network can exploit the aforementioned parameters is related to the network architecture. Regarding the network architecture, the co-located networks can be either under the control of a central coordinator or can operate independently.

According to the coordinated approach, the identification of the participating networks and the collection of traffic information can be easy as the coordinator can directly communicate with each network. On the other hand, the existence of a coordinator increases the complexity of the network. Additionally, there always might be other networks in the neighbourhood that do not belong to the coordination scheme. Modifications to the wireless protocols are required in order to render each technology capable of communicating 


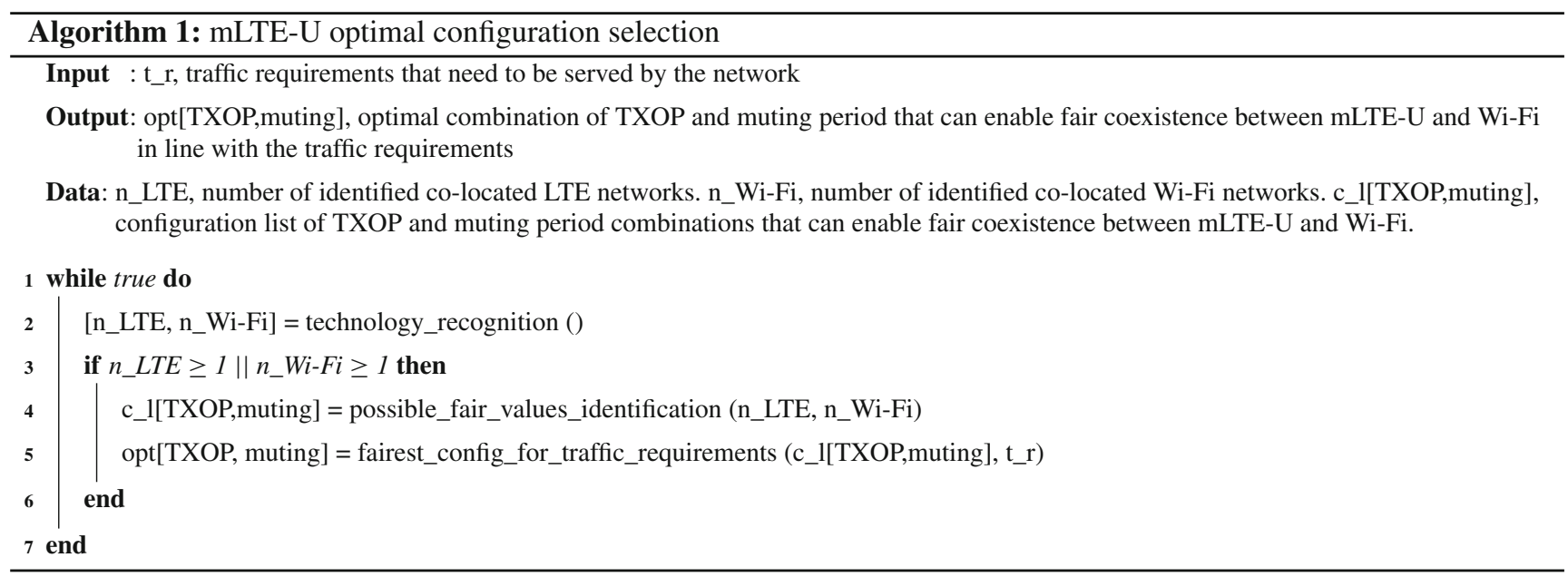

such type of information to the coordinator. The coordinator needs a careful design in order to be able to communicate with different technologies, collect and manage the required information. In such an ideal scenario, the coordinator will be responsible to tune the mLTE-U parameters in order to ensure that each network is able to achieve the required throughput. On the other hand, a non-coordinated approach is more realistic, as every network can be deployed arbitrarily. Such an approach requires lower complexity regarding the overall network architecture as each network operates independently. On the contrary, each network must be responsible to collect the information that is required in order to decide the appropriate configuration that enables fair coexistence with the co-located networks. Wireless technology recognition techniques $[7,30]$ are required to identify the amount and type of the wireless technologies that are in the proximity of each other. Based on this information, each mLTE-U network can decide the combinations of TXOP and the muting period that can offer the proportional fair throughput.

The discovery of the TXOP and muting period configurations that offer fair coexistence requires careful design. As a first approach, a heuristic technique can be used. According to such a technique, the eNB can try different configurations attempting to find the ones that offer a performance (e.g. throughput) that approaches its target. When a combination of TXOP and muting period is found, the eNB can evaluate other configurations by using neighbouring values for both TXOP and muting period. As it has been observed by the simulation result, neighbouring configuration values are more possible to offer fair coexistence. Hence, for instance if a TXOP duration of $4 \mathrm{~ms}$ followed by a muting period of 8 $\mathrm{ms}$ is a possible configuration, then a next possible combination could be a TXOP of $5 \mathrm{~ms}$ followed by a muting period of $8 \mathrm{~ms}$. As in every learning technique, this method requires a convergence time to identify the desired configurations. In the beginning, the system can operate in acceptable bounds but as the time passes the considered heuristic algorithm approaches to the optimal configuration values in reasonable time. The complexity is in line with our previous work [31], in which two heuristic algorithms for joint power assignment and resource allocation in femtocells are evaluated and optimized in order to achieve the optimal solution in short time. The design of an algorithm that determines the configurations that can offer fair coexistence in an optimal way will be further examined in our future work.

Based on the identified combinations of TXOP and muting period that offer fair coexistence for a specific topology and according to the traffic requirements that must be satisfied, the network can select the optimal configuration that serves them better. For instance, in case of voice traffic (AC_VO), the network must choose a configuration that offers a short muting period and a long TXOP. On the contrast, a network that must serve best-effort traffic (AC_BE) can choose a configuration with longer muting period and shorter TXOP.

Algorithm 1 presents the complete procedure as it is described above and is required by an independent mLTE$\mathrm{U}$ network to select an optimal configuration that enables fair coexistence with the co-located LTE or Wi-Fi networks. The algorithm takes as input the traffic requirements that the mLTE-U network has to serve. Then, periodically it performs a technology recognition in order to identify potential co-located LTE or Wi-Fi networks. Based on the discovered networks, the algorithm determines the possible values (TXOP and muting period) that can provide fair coexistence (e.g. using a heuristic technique). Finally, based on the traffic requirements, it selects the optimal parameters that enable fair spectrum sharing. Further study and optimization of the techniques that can identify an optimal mLTE-U configuration based on different topologies and traffic requirements for both LTE and Wi-Fi will be investigated in our future work. 


\section{Conclusions and future work}

This article proposes a new coexistence scheme that can enable a fair coexistence of LTE-U and Wi-Fi. As it is discussed, a fair coexistence can give to the participating networks opportunities to achieve equal performance in a technology-agnostic manner. The proposed coexistence scheme named mLTE-U, requires a CCA procedure before each mLTE-U transmission. When the CCA mechanism indicates the channel as idle, then the mLTE-U performs a transmission burst of variable duration followed by a muting period of variable duration. The muting period can give further transmission opportunities to coexisting Wi-Fi networks. The proposed mLTE-U scheme and the provided coexistence with Wi-Fi and other mLTE-U networks is evaluated in different scenarios of high interest. These scenarios include different mLTE-U and Wi-Fi network densities, as well as static and moving end-devices. Furthermore, we discuss the procedure according to which an mLTE-U network can select the parameters that can offer the required fair coexistence in a technology-agnostic manner, based on the number of participating networks and the traffic requirements that must be satisfied. The simulation results show that the proper configuration of mLTE-U according to the number of co-located networks can enable fair and harmonious coexistence in unlicensed spectrum.

In the near future, we will further investigate and analyse techniques towards the optimal selection of the mLTE-U parameters that can enable fair coexistence with co-located wireless technologies.

Acknowledgements The research leading to this article has received funding from the European FP7 Program under grant agreement 612050 (FLEX project) and from the European H2020 Programs under grant agreement 688116 (eWINE project) and 645274 (WiSHFUL project).

Open Access This article is distributed under the terms of the Creative Commons Attribution 4.0 International License (http://creativecomm ons.org/licenses/by/4.0/), which permits unrestricted use, distribution, and reproduction in any medium, provided you give appropriate credit to the original author(s) and the source, provide a link to the Creative Commons license, and indicate if changes were made.

\section{References}

1. Qualcomm (2012). Rising to meet the 1000x mobile data challenge.

2. 3GPP TS 36.213 V13.3.0. Evolved Universal Terrestrial Radio Access (E-UTRA); Physical layer procedures. Release 13

3. Qualcomm. (2015). Making the Best Use of Unlicensed Spectrum for $1000 \mathrm{x}$.

4. 3GPP News. (2015). Evolution of LTE in release 13. http://www. 3gpp.org/news-events/3gpp-news/1628-rel13.

5. MulteFire Alliance. http://www.multefire.org/.

6. IEEE Standard 802.11. (2012). Wireless LAN medium access control (MAC) and physical layer (PHY) specifications.
7. Rajab, S. A., Balid, W., Al Kalaa, M. O., \& Refai, H. H. Energy detection and machine learning for the identification of wireless MAC technologies. In 2015 international wireless communications and mobile computing conference (IWCMC) (pp. 1440-1446). https://doi.org/10.1109/IWCMC.2015.7289294.

8. 3GPP TS 36.211. Evolved Universal Terrestrial Radio Access (EUTRA); Physical Channels and Modulation. Release 12

9. Maglogiannis, V., Naudts, D., Willemen, P., \& Moerman, I. (2016). Impact of LTE operating in unlicensed spectrum on Wi-Fi using real equipment. In IEEE global telecommunications conference (GLOBECOM) (pp. 1-6). Washington, DC, USA. https://doi.org/ 10.1109/GLOCOM.2016.7841884.

10. iLab.t IMEC LTE testbed. http://doc.ilabt.iminds.be/ilabtdocumentation/.

11. Jian, Y., Shih, C. F., Krishnaswamy, B., \& Sivakumar, R. (2015). Coexistence of Wi-Fi and LAA-LTE: Experimental evaluation, analysis and insights. In 2015 IEEE international conference on communication workshop (ICCW) (pp. 2325-2331). https://doi. org/10.1109/ICCW.2015.7247528.

12. Sagari, S., Seskar, I., \& Raychaudhuri, D. Modeling the coexistence of LTE and WiFi heterogeneous networks in dense deployment scenarios. In 2015 IEEE international conference on communication workshop (ICCW) (pp. 2301-2306). https://doi.org/10.1109/ ICCW.2015.7247524.

13. Ratasuk, R., Uusitalo, M. A., Mangalvedhe, N., Sorri, A., Iraji, S., Wijting, C., \& Ghosh, A. (2012). License-exempt LTE deployment in heterogeneous network. In IEEE international symposium on wireless communication systems (ISWCS) (pp. 246-250). https:// doi.org/10.1109/ISWCS.2012.6328367.

14. Nihtil, T., Tykhomyrov, V., Alanen, O., Uusitalo, M. A., Sorri, A., Moisio, M., Iraji, S., Ratasuk, R., \& Mangalvedhe, N. (2013). System performance of LTE and IEEE 802.11 coexisting on a shared frequency band. In IEEE wireless communications and networking conference (WCNC) (pp. 1038-1043). https://doi.org/10.1109/ WCNC.2013.6554707.

15. Almeida, E., Cavalcante, A. M., Paiva, R. C., Chaves, F. S., Abinader, F. M., Vieira, R. D., Choudhury, S., Tuomaala, E., \& Doppler, K. (2013). Enabling LTE/WiFi coexistence by LTE blank subframe allocation. In IEEE international conference on communications (ICC) (pp. 5083-5088). https://doi.org/10.1109/ICC. 2013.6655388.

16. 3GPP TR 36.808. Evolved Universal Terrestrial Radio Access (EUTRA); Carrier Aggregation; Base Station (BS) radio transmission and reception. Release 10

17. Kwon, H. J., Jeon, J., Bhorkar, A., Ye, Q., Harada, H., Jiang, Y., Liu, L., Nagata, S., Ng, B. L., Novlan, T., Oh, J., \& Yi, W. (2016). Licensed-assisted access to unlicensed spectrum in LTE Release 13. In IEEE communications magazine (Issue:99, pp. 2-8). https:// doi.org/10.1109/MCOM.2016.1500698CM.

18. Mukherjee, A., Cheng, J. F., Falahati, S., Koorapaty, H., Karaki, R., Falconetti, L., et al. (2016). Licensed-assisted access LTE: Coexistence with IEEE 802.11 and the evolution toward 5G. IEEE Communications Magazine, 54(6), 50-57. https://doi.org/10.1109/ MCOM.2016.7497766.

19. Al-Dulaimi, A., Al-Rubaye, S., Ni, Q., \& Sousa, E. (2015). 5G communications race: Pursuit of more capacity triggers LTE in unlicensed band. IEEE Vehicular Technology Magazine, x, 43-51. https://doi.org/10.1109/MVT.2014.2380631.

20. Kim, C. K., Yang, C. S., \& Kang, C. G. (2016). Adaptive listenbefore-talk (LBT) scheme for LTE and Wi-Fi systems coexisting in unlicensed band. In 13th IEEE annual consumer communications and networking conference (CCNC) (pp. 1-6). https://doi.org/10. 1109/CCNC.2016.7444845.

21. Mushunuri, V., Panigrahi, B., Rath, H. K., \& Simha, A. (2017). Fair and efficient listen before talk (LBT) technique for LTE licensed assisted access (LAA) networks. In IEEE 31 st international confer- 
ence on advanced information networking and applications (AINA) (pp. 39-45). https://doi.org/10.1109/AINA.2017.135.

22. Hao, F., Yongyu, C., Li, H., Zhang, J., \& Quan, W. (2016). Contention window size adaptation algorithm for LAA-LTE in unlicensed band. In International symposium on wireless communications systems (ISWCS) (pp. 476-480). https://doi.org/10.1109/ ISWCS.2016.7600951.

23. Li, H., Chang, Y., Hao, F., Men, A., Zhang, J., \& Quan, W. (2016). Study on dynamic channel switch in license-assisted-access based on listen-before-talk. In International symposium on wireless communication systems (ISWCS) (pp. 506-510). https://doi.org/10. 1109/ISWCS.2016.7600957.

24. Bhorkar, A., Ibars, C., Papathanassiou, A., \& Zong, P. (2015). Medium access design for LTE in unlicensed band. In Wireless communications and networking conference workshops (WCNCW) (pp. 369-373). https://doi.org/10.1109/WCNCW.2015.7122583.

25. Maglogiannis, V., Naudts, D., Shahid, A., Giannoulis, S., Laermans, E., \& Moerman, I. (2017). Cooperation techniques between LTE in unlicensed spectrum and Wi-Fi towards fair spectral efficiency. Sensors, 17(9), 1994. https://doi.org/10.3390/s17091994.

26. Chen, B., Chen, J., Gao, Y., \& Zhang, J. (2017). Coexistence of LTE-LAA and Wi-Fi on $5 \mathrm{GHz}$ with corresponding deployment scenarios: A survey. IEEE Communications Surveys and Tutorials, 19(1), 7-32. https://doi.org/10.1109/COMST.2016.2593666.

27. Sharon, O., \& Alpert, Y. (2014). MAC level Throughput comparison: 802.11ac vs. 802.11n. Physical Communications, 12, 33-49. https://doi.org/10.1016/j.phycom.2014.01.007.

28. Saif, A., Othman, M., Subramaniam, S., \& Abdul Hamid, N. A. (2011). Frame aggregation in wireless networks: Techniques and issues. IETE Technical Review, 28(4), 336-350. https://doi.org/10. 4103/0256-4602.83554

29. 3GPP TS 36.300. Evolved Universal Terrestrial Radio Access (EUTRA) and Evolved Universal Terrestrial Radio Access Network (E-UTRAN); Overall description; Stage 2. Release 10

30. Gardner, W. A. (1988). Signal interception: A unifying theoretical framework for feature detection. IEEE Transactions on Communications, 36(8), 897-906. https://doi.org/10.1109/26.3769.

31. Shahid, A., Aslam, S., \& Lee, K. G. (2013). A decentralized heuristic approach towards resource allocation in femtocell networks. Entropy, 15(7), 2524-2547. https://doi.org/10.3390/e15072524.

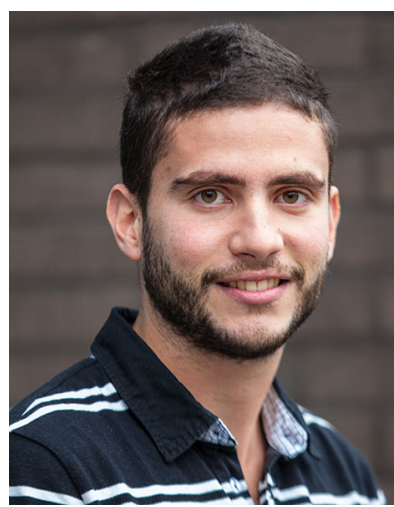

Vasilis Maglogiannis received his B.Eng degree in Computer Engineering and his M.Sc in Science and Technology of Computers, Telecommunications and Networks from University of Thessaly, Greece in 2012 and 2014 respectively. In 2014, he joined the IDLab of the Department of Information Technology, University of Ghent-imec, Belgium. He is currently pursuing his Ph.D. degree in the faculty of Engineering and Architecture at University of Ghent, Belgium. His main research interests include mobile and wireless networks, 5G, LTE, 802.11, 802.15.4 and wireless sensor networks. He has experience in working on national, FP7 and H2020 European projects.

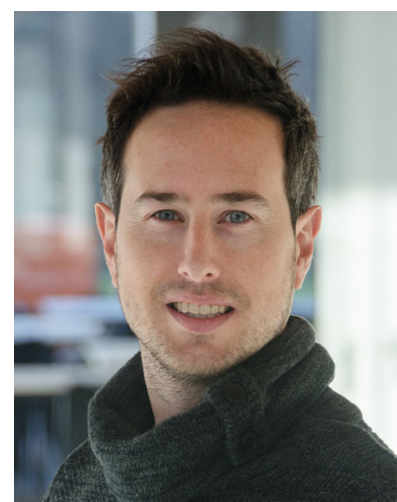

Dries Naudts received his M.Sc degree in Computer Science from the Ghent University, Belgium in 2001. He started as a software engineer at Kresoft and Nisus. In April 2005, he joined the IDLab research group of the Department of Information Technology, University of Ghent-imec, Belgium, where he works as a senior researcher. His research interests are in mobile networks, 5G, LTE, 802.11 and C-ITS. He works on several national, international R\&D, FP7 and H2020 European projects.

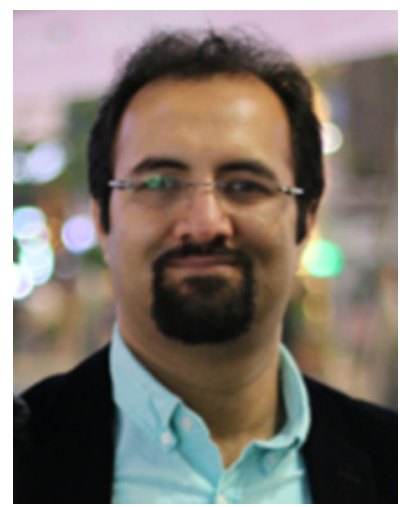

Adnan Shahid received the B.Eng. and the M.Eng. degrees in computer engineering with wireless communication specialization from the University of Engineering and Technology, Taxila, Pakistan in 2006 and 2010, respectively, and the Ph.D. degree in information and communication engineering from the Sejong University, South Korea in 2015. He is currently working as a Postdoctoral/Senior Researcher at IDLab, Department of Information Technology, University of Ghent-imec, Belgium, where he is involved in several European research projects including eWINE, WiSHFUL, etc and other national projects including SAMURAI, IDEAL-IOT, MUSCLE-IOT, etc. In addition, he is also technically coordinating the eWINE project. From Sep 2015-Jun 2016, he was with the Department of Computer Engineering, Taif University, Saudi Arabia. From Mar 2015-Aug 2015, he worked as a Postdoc Researcher at Yonsei University, South Korea. He was also the recipient of the prestigious BK21 plus Postdoc and Research Professor positions at Yonsei University, South Korea. He is a senior member of IEEE and actively involved in various research activities. $\mathrm{He}$ is also serving as an Associate Editor at IEEE Access Journal and Journal of Networks and Computer Application, Elsevier. He has over 30 research papers in well-known venues at journals, conferences, patents, etc. His research interests includes the next generation wireless communication and networks with prime focus on resource management, interference management, cross-layer optimization, selforganizing networks, small cell networks, device to device communications, machine to machine communications, Sug-GHz technologies, $5 \mathrm{G}$ wireless communications, machine learning, etc. 


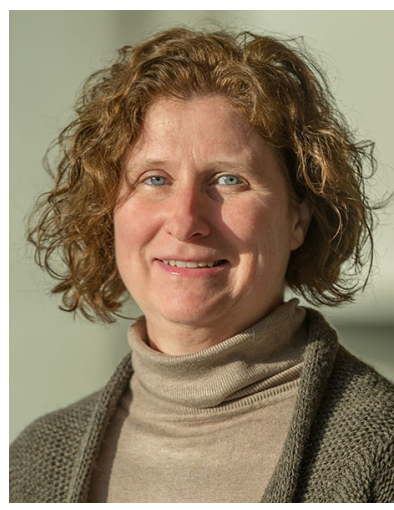

Ingrid Moerman received her degree in Electrical Engineering (1987) and the Ph.D. degree (1992) from the Ghent University, where she became a part-time professor in 2000. She is a staff member at IDLab, a core research group of imec with research activities embedded in Ghent University and University of Antwerp. Ingrid Moerman is coordinating the research activities on mobile and wireless networking, and she is leading a research team of about 30 members at IDLab-Ghent University. Her main research interests include: Internet of Things, Low Power Wide Area Networks (LPWAN), High-density wireless access networks, collaborative and cooperative networks, intelligent cognitive radio networks, real-time software defined radio, flexible hardware/software architectures for radio/network control and management, and experimentally-supported research. Ingrid Moerman has a longstanding experience in running and coordinating national and $\mathrm{EU}$ research funded projects. At the European level, Ingrid Moerman is in particular very active in the Future Networks research area, where she has coordinated and is coordinating several FP7/H2020 projects (CREW, WiSHFUL, eWINE, ORCA) and participating in other projects (Fed4FIRE, FORGE, FLEX, Flex5Gware). Ingrid Moerman is author or co-author of more than 700 publications in international journals or conference proceedings. 